\title{
PATH AND DIRECTIONALITY DISCOVERY IN INDIVIDUAL DYNAMIC MODELS: A REGULARIZED UNIFIED STRUCTURAL EQUATION MODELING APPROACH FOR HYBRID VECTOR AUTOREGRESSION
}

\author{
AI Ye \\ Kathleen Gates \\ Teague Henry \\ LAN LUO
}

L. L. THURSTONE PSYCHOMETRIC LAB

DEPARTMENT OF PSYCHOLOGY AND NEUROSCIENCE

UNIVERSITY OF NORTH CAROLINA AT CHAPEL HILL 


\begin{abstract}
There recently has been growing interest in the study of psychological and neurological processes at an individual level. One goal in such endeavors is to construct person-specific dynamic assessments using time series techniques such as Vector Autoregressive (VAR) models. However, two problems exist with current VAR specifications: 1) VAR models are restricted in that contemporaneous relations are typically modeled either as undirected relations among residuals or directed relations among observed variables, but not both; 2) current estimation frameworks are limited by the reliance on stepwise model building procedures. This study adopts a new modeling approach. We first extended the current unified SEM (uSEM) framework, a widely used structural VAR model, to a hybrid representation (i.e., "huSEM") to include both undirected and directed contemporaneous effects, and then replaced the stepwise modeling with a LASSO-type regularization for a global search of the optimal sparse model. Our simulation study showed that regularized huSEM performed uniformly the best over alternative VAR representations and/or modeling approaches, with respect to accurately recovering the presence and directionality of hybrid relations and reliably removing false relations when the data are generated to have two types of contemporaneous relations. The present study to our knowledge is the first application of the recently developed regularized SEM technique to the estimation of huSEM, which points to a promising future for statistical learning in psychometric models.
\end{abstract}

Key words: Time Series Data; Structural VAR; Hybrid VAR; Contemporaneous Relations; Unified SEM; Graphical VAR; Regularized SEM 


\section{INTRODUCTION}

There recently has been growing interest in the study of psychological processes at the individual level, partly due to the increasing availability of time series data in psychological and neurological science. Time series data here refers to data with many measurements over time, ideally over 60 per person. Examples include ecological momentary assessment and experience sampling methods such as daily dairy (e.g., Myin-Germeys et al., 2009; Wright et al., 2015), or neurological measurements obtained from functional neuroimaging (Price et al., 2017). One common goal is to construct person-specific dynamic assessments based on intraindividual variability (within-person variability; e.g., Bringmann et al., 2013; Bringmann et al., 2015; Ram \& Gerstorf, 2009; Wigman et al., 2015). Arriving at person-specific time series models and depicting them as networks can aid clinicians in developing treatment plans for patients (Fisher \& Boswell, 2016) and also help in making valid inferences in research studies. For example, identifying individual-level nuances can aid in the understanding of heterogeneity in symptom representation among clinical patients (Wright et al., 2015) and differences in cognitive processes (Nichols et al., 2014). Person-specific intraindividual variability research has been applied to widespread psychological and neurological contexts such as language learning (Yang et al., 2015), the interplay of personality disorder symptoms (Wright et al., 2015), and traumatic brain injury (Hillary et al., 2015), to name a few. In this paper, we present a novel data-driven modeling approach to circumvent the current limitations seen in some popular methods. We first extended the current unified SEM (uSEM) framework, a widely used structural VAR model, to a hybrid representation (i.e., "huSEM") that includes both undirected and directed contemporaneous effects. Next, we apply a LASSO-type regularization for a global search to arrive at the optimal sparse model. Importantly, our approach expands the analytic capabilities of intraindividual variability research by allowing for a larger search space than currently available methods. By doing so, we allow for more reliable interpretation of the resulting models.

Intraindividual variability is commonly investigated using multivariate time series methods. One framework is the Vector Autoregressive (VAR) model that assesses the dependency between multivariate repeated measures across time using linear lagged relations. However, intraindividual variability researchers face a conundrum when using the VAR models in practice. The issue is the manner with which contemporaneous effects, or relations among variables that appear to occur instantaneously, are considered. Such effects are common among psychological variables and often quantified after taking into account any lagged relations. Widely-used VAR specifications are restricted in that contemporaneous relations are modeled either as structural (directed) types of associations among observed variables or as residual covariances (bidirectional or undirected), but not both. However, the way in which the relations are modeled carry substantive interpretations that differ theoretically. In an effort to ensure that the resulting model will be estimable (i.e. identified), modeling approaches typically require that the researcher choose to model all contemporaneous relations as either entirely directed or undirected. It is likely that both types of contemporaneous relations are present for a given individual's dynamic process. For example, we might expect that daily stress has a direct (perhaps causal) influence on negative affect on that same day, whereas any relation between fatigue and energy levels may be caused by something external to the system of variables acquired and thus exhibited as a covariance among residuals. An appropriate model should have the flexibility to disclose mixed types of contemporaneous relations should 
they exist. Only allowing for one type of specification has the potential to result in either missing relations that do exist or introducing false positives when the wrong expression was chosen or in reality both exist.

This limitation has been discussed previously, with the general expression that allows for both types of contemporaneous relations provided by Lütkepohl (2005, who termed it the full VAR) and by Molenaar (2019, 2016, who termed it the hybrid VAR). The hybrid VAR approach for individual-level analyses has not been fully evaluated with simulation studies or used in any empirical study in psychology. One reason why this has not been explored is the challenge of arriving at patterns of relations that are identifiable and can be estimated. Without inducing sparsity in some way the a hybrid VAR would be saturated. Therefore, when fitting a VAR model to data in practice, one needs to arrive at a sparse, identifiable model, that is, one with a unique solution for the remaining nonzero parameters. Ambiguity exists because a priori theory to guide researchers through the discovery of true sparseness is often scant. As Lütkepohl (2005) describes, it is exceedingly difficult to define rules for identifiability. The question becomes: How to determine the identifiable model that best corresponds to the underlying causal structure of the data? One available option involves a step-wise searching schema whereby relations are added in a forward-search, which we will discuss in detail in later section. A drawback of this approach is that the final model is heavily influenced by relations that are added early in the search procedure.

Inspired by recent developments of statistical learning technique in psychometric models (Huang et al., 2017; Jacobucci et al., 2016; Lane, 2017), this article presents a novel modeling framework for a flexible VAR approach that estimates a sparse set of both undirected and directed contemporaneous effects. We build from the unified SEM (Gates et al., 2010; Kim et al., 2007) approach which estimates VAR models from within an SEM framework. The uSEM specification only allows for directed relations among observed variables. We expand this to include bidirectional relations among residuals in the search space (Molenaar, 2017). The approach introduced here, called regularized hybrid unified SEM (or regularized huSEM, or simply, Reg-huSEM), simultaneously performs a global search and estimation for the optimal sparse hybrid VAR model using regularization approaches. We evaluate currently popular VAR specification approaches using simulation studies - specifically, graphical VAR (Epskamp et al., 2018) which allows solely for partial correlation among relations and unified SEM (Gates et al., 2010), which allows solely for directed relations among observed variables - as well a the huSEM. Certainly, our ultimate interest is to evaluate the performance of regularization under the hybrid VAR model that allows for both types of contemporaneous relations. Of greatest interest is the ability to reach an optimal model that recovers precisely the presence and directionality of only the true relations.

The remainder of the paper is structured as follows. We first provide the technical background of major VAR specifications. Next, we review and compare model discovery and estimation techniques under each VAR model. We then describe the rationale and implementation of the new model discovery and estimation framework into the current VAR specifications. This is followed by a simulation study to compare the performance of the existing and newly proposed modeling framework under conditions that mimic empirical settings. To close, we discuss interpretations of hybrid relations in VAR models, as well as the contribution of our proposal to the development of exploratory SEM research. 


\section{TECHNICAL BACKGROUND}

We begin with some definitions necessary for understanding VAR models. In some cases a variable measured at a given time can explain variability in a future observation of itself; this relation is a lagged relation often referred to as an "autoregressive (AR) effect". In a multivariate case, a lagged effect from one variable to another at a later measurement is referred to as a "cross-lagged effect". They are the source for observed temporal dependency among repeated measures across time. When a cross-lagged effect is significant after taking into account the AR effect it is said that Granger causality (Granger, 1969) takes place. Granger causality is defined as follows: variable $X$ is said to Granger cause variable $Y$ if $Y$ values are better predicted with values of $X$ (either contemporaneously or lagged) than with past values of $Y$ alone (Geweke, 1982; Granger, 1969; Lütkepohl, 2005). In either case the

direction of the effect (i.e. $X \stackrel{G C}{\longrightarrow} Y$ or $Y \stackrel{G C}{\longrightarrow} X$ ) discloses the information flow, with the possibility of both variables Granger causing each other.

In multivariate intraindividual variability approaches, Granger causal relations occurring at the same measurement is referred to as contemporaneous or instantananeous effects. Contemporaneous relations exist in empirical data due to subsampling. This occurs in functional MRI since data is collected at a rate far slower than the neuronal processes of interest, and similarly in daily diary studies where the data collection is at a slower rate than the speed in which constructs of interest (e.g., emotions) change. Contemporaneous relations are similar to cross-sectional (or interpersonal variability analysis in interpretation. However, contemporaneous relations in interindividual variability are usually modeled upon the assumption that observations are independent, while in intra-individual variability the relation is conditional upon the AR and cross-lagged relations. This is a key difference between cross-sectional and time series modeling - ordering of observations matters in the latter approach. Just as in inter-individual variability, contemporaneous relations in intra-individual variability can be undirected or directed. With intra-individual variability, an instantaneous directed effect is likely to be observed when a causal effect unfolds within a shorter span than the size of lag interval. We will return to the interpretation of the directed contemporaneous relations with further detail in the discussion.

\section{Traditional VAR}

The VAR model (Hamilton, 1994; Shumway \& Stoffer, 2017) is a traditional time series technique for modeling person-specific processes using time series data. Let $Y_{t}=\left[y_{1 t}, y_{2 t}, \ldots, y_{p t}\right]^{\prime}$ be a vector of a $p$-variate time series, $p>1$, at a given time point $t$, with $t=1, \ldots, T$. Suppose $Y_{t}$ can be represented by a stationary linear time series (i.e., having a constant means and covariance function), defined by the following VAR model with lag order- $m, \operatorname{VAR}(\mathrm{m})$ :

$$
Y_{t}=\boldsymbol{\Phi}_{\mathbf{1}} Y_{t-1}+\mathbf{\Phi}_{\mathbf{2}} Y_{t-2}+\ldots+\boldsymbol{\Phi}_{\boldsymbol{m}} Y_{t-m}+\varepsilon_{t}, \varepsilon_{t} \sim N_{p}(\mathbf{0}, \boldsymbol{\Theta})
$$

where the sequence of $(p, p)$ dimensioned $\Phi_{k}$ matrices are the lagged coefficient matrices at order $k, k=1, \ldots, m$, with diagonal elements the $\mathrm{AR}(k)$ regression coefficients and the off-diagonal cross-lagged coefficients. For simplicity, the intercept is omitted here. The coefficients are assumed to be time invariant. Further, there is no auto or cross-lagged 
dependency in the error term $\varepsilon_{t}$. i.e., the temporal covariance at lag $u$, $\Theta_{u}=\operatorname{cov}\left[\varepsilon_{t}, \varepsilon_{t-u}^{\prime}\right]=0 \forall u \in 1 \ldots \infty$. This zero-mean $p$-variate $\varepsilon_{t}$ may be correlated contemporaneously.

The simplest form is the first-order standard VAR model, or lag-1 VAR, or simply $\operatorname{VAR}(1)$. VAR(1) estimates temporal relations of only consecutive measurements:

$$
Y_{t}=\boldsymbol{\Phi}_{1} Y_{t-1}+\varepsilon_{t}, \varepsilon_{t} \sim N_{p}(\mathbf{0}, \boldsymbol{\Theta})
$$

VAR(1) model assumes a "memoryless" (i.e. Markov) process in which all previous information useful to predict the current values of $Y_{t}$ is contained in $Y_{t-1}$. For an illustration of the new modeling framework, all the VAR models discussed in the current study are VAR(1) processes. This also follows the use of such personalized models in practice (e.g., Fisher, 2015; Wright et al., 2015). The approaches discussed here generalize to higher-order VAR models but is outside the scope of the present paper.

\section{Structural VAR}

As pointed out by Econometricians (e.g., Shapiro \& Watson, 1988; Sims, 1981), VAR is a "reduced form" of a more general class of models referred to as the Structural VAR (SVAR; Chen et al., 2011). Instead of only identifying AR and cross-lagged coefficients, SVAR incorporates directed contemporaneous associations. A fully saturated SVAR model includes free parameters for all possible direct effects among observed variables and among residuals. However, the fully saturated model is not identifiable. In practice, restriction is imposed to reduce it to a testable model, typically with only one form of contemporaneous relations allowed and the lower diagonal of the corresponding matrix estimated (Lütkepohl, 2005). The uSEM approach represents a reformulation of a type of SVAR model where the directed contemporaneous relations occur among the observed variables. The USEM is written as (Gates et al., 2010):

$$
Y_{t}=\boldsymbol{A} Y_{t}+\boldsymbol{\Phi}_{1}^{*} Y_{t-1}+\zeta_{t}, \zeta_{t} \sim N(\mathbf{0}, \mathbf{\Psi})
$$

The $p \times p \boldsymbol{A}$ matrix contains the contemporaneous estimates for relations among the component series of $Y_{t}$, with independent white noise $\zeta_{t}$ (or "innovations") having a diagonal covariance matrix $\boldsymbol{\Psi}$. Two coefficients, $a_{i j}$ and $a_{j i}$, captures the presence and directionality of the relation between two given variables at time $y_{t i}$ and $y_{t j}$, with $a_{i j}$ representing the opposite directionality of $a_{j i}$. Note that estimates in the lagged relation matrix now differ: $\Phi \neq \Phi^{*}$

\section{A Synthesis: the Hybrid VAR}

VAR-based models estimate the directed lagged relations first and then assess the (contemporaneous) covariance among residuals as a second step. The lagged relations thus get priority as these are discovered and estimated without any possibility of conditioning on potential contemporaneous relations. Should they exist, failure to include the contemporaneous directed relations will influence the estimates obtained in VAR. Additionally, 
contemporaneous directed relations will often surface as lagged relations when modeled as a VAR (Gates et al., 2010), which can lead to inaccurate inferences. From an interpretation standpoint, one can consider a bi-directional instantaneous connections as a contemporaneous non-causal mutual dependence. In this case, the relation among two given variables is thought to occur due to the influence of something outside the system.

On the other end of the spectrum, only uni-directional contemporaneous relations are considered in the uSEM approaches. One variable can contemporaneously explain the variability in another alongside the lagged relations. In this case, the errors are assumed to have no contemporaneous relations among them. The interpretation here is that all variables in the model are endogenous and as such can be explained by other variables within the system. This might be too strong an assumption in some cases, such as when two variables are related through an latent or observed variable that are not included in the model. The interpretation of these directed contemporaneous relations is that if we have knowledge of the independent variable, we can explain a significant degree of variability in the target variable contemporaneously. We refer to such contemporaneous relations as instantaneous causal structural path.

In practice, researchers tend to either conduct the VAR model with bidirectional or undirected relations among residuals (e.g., graphical VAR, Epskamp \& Fried, 2016) or the uSEM with directed contemporaneous relations among observed variables. The decision of which to use carries with it assumptions that relate to how the data at hand are thought to be generated and how to interpret results. As just outlined, the interpretation of contemporaneous relations in VAR and uSEM models differs. However, it is possible that the underlying relations in empirical settings are mixed, with some instantaneous associations being best captured as non-causal dependencies and other relations in the data aligning with causal structural paths. When this is the case, neither uSEM nor VAR with contemporaneous residual relations would be appropriate. Molenaar $(2017,2019)$ promoted the use of a hybrid $V A R$ approach which combines USEM and traditional VAR into one model. The model search space of the hybrid VAR incorporates a contemporaneous relation as either a structural relation among observed variables (as in USEM) or a covariance among residuals (as in VAR), allowing for the flexibility in the types of contemporaneous relations. This hybrid VAR is a special case of the full SVAR. To formally introduce the hybrid VAR, we turn to the details of the modeling framework used here, which is based within the SEM framework.

\section{MODELING FRAMEWORKS}

\section{Estimating VAR under the extended Gaussian Graphical Modeling}

A variant of the VAR model is often performed by use of a graphical representation under the framework of Gaussian Graphical Modeling (GGM; Abegaz \& Wit, 2013; Eichler, 2005; Wild et al., 2010). At its core, the GGM attempts to model the relations between variables (also called "nodes") using a sparse set of partial correlations, which is achieved by applying regularization to a given inverse covariance matrix under the assumption that the data is distributed as a multivariate normal distribution (Lauritzen, 1996). Meaningful bivariate relations are represented as nonzero edges between nodes while a lack of edge suggests independence after conditioning on all other variables in the system. Since conditional 
independence among psychological variables is more common than is marginal independence (Meehl, 1990), it is more likely to attain a parsimonious model by turning the estimation of marginal relations to conditional relations (Wild et al., 2010). GGM has been extended to what is called graphical VAR (gVAR; Epskamp et al., 2018; Wild et al., 2010): at the lag-0 level, a weighted, undirected contemporaneous network, called partial contemporaneous correlations (PCC), is drawn following the general GGM fashion on the observed variables. In the meantime, the approach incorporates the temporal ordering dependency by generating a weighted, directed temporal network, termed partial directed correlation (PDC). This is formed at the lag-1 level of gVAR (Eichler, 2005; Wild et al., 2010).

Obtaining a sparse PCC and PDC results involves three major steps. First, the temporal relations in PDC are obtained via a standardization on $\phi$ elements, representing the linear lagged relationship between $y_{i, t}$ and $y_{j, t-1}$, net the linear effect of all other variables at $t-1$ that are included in the model. This comprises the VAR portion of the model. Next, a precision matrix is computed by taking the inverse of covariance matrix $\Theta$ in Equation 2 . Partial correlations in PCC nework are obtained via a standardization on the elements of precision matrix $\boldsymbol{\kappa}$ derived by $\operatorname{PCC}\left(Y_{i, t}, Y_{j, t}\right)=\operatorname{Cov}\left(Y_{i, t}, Y_{j, t} \mid \boldsymbol{y}_{-(i, j)}\right)=-\frac{\kappa_{i, j}}{\sqrt{\kappa_{i i}} \sqrt{\kappa_{j j}}}$, where $\kappa_{i, j}$ denotes an element in , $\boldsymbol{y}_{-(\boldsymbol{i}, \boldsymbol{j})}$ denotes all variables subtracting variable $i$ and $j$, hence PCC represents the correlation between $i$ and $j$ at time $t$, after partialing out the linear effects of all other variables and any lagged relations. Finally, small partial correlations at both levels are first forced to zero by thresholding rules The remaining parameters are then regularized and shrink towards zero (more regularization background will be discussed in later sections).

\section{Estimating SVAR under Unified Structural Equation Modeling}

Structural equation modeling (SEM; Bollen, 1989) is a suitable estimation framework for SVAR models since time series estimates obtained via SEM are similar to estimates obtained using more traditional approaches such as the Kalman Filter (Chow et al., 2010; Hamaker et al., 2002). Conventional SEM has been extended to unified SEM modeling (uSEM; Gates et al., 2011; Kim et al., 2007) for time series data. As the name explains, uSEM "unifies" temporal ordering dependency and contemporaneous associations among observed variables. One advantage of specifying SVAR in USEM is that it solves the issue of "nonunique solution" by using a stepwise procedure for selecting paths Molenaar (2019).

We can write Model 3 using conventional SEM notation (Gates et al., 2017):

$$
\boldsymbol{Y}=\boldsymbol{B} \boldsymbol{Y}+\boldsymbol{\zeta}, \boldsymbol{\zeta} \sim N(\mathbf{0}, \boldsymbol{\Psi})
$$

where $\boldsymbol{Y}=\left[\boldsymbol{Y}_{\boldsymbol{t}-\mathbf{1}}, \boldsymbol{Y}_{\boldsymbol{t}}\right]$ is a $2 p x T$ matrix. That is, the observations of variables are time-embedded by appending the data at $t-1$ to the data at $t$. The data are thus expanded to two consecutive time points $t-1$ and $t$, so time series vector of lagged (exogenous) variables and those of contemporaneous (endogenous) variables are appended horizontally. This requires that the $\boldsymbol{\zeta}$ error vector also be extended, as well as each of the corresponding matrices. The contemporaneous and lagged coefficients collapse into a single $2 p \times 2 p \boldsymbol{B}$ regression coefficient matrix: 


$$
\boldsymbol{B}=\left(\begin{array}{cccccccccc}
0 & \cdots & \cdots & \cdots & 0 & 0 & \cdots & \cdots & \cdots & 0 \\
\vdots & \ddots & & & \vdots & \vdots & \ddots & & & \vdots \\
\vdots & & \ddots & & \vdots & \vdots & & \ddots & & \vdots \\
\vdots & & & \ddots & \vdots & \vdots & & & \ddots & \vdots \\
0 & \cdots & \cdots & \cdots & 0 & 0 & \cdots & \cdots & \cdots & 0 \\
\phi_{11}^{*} & \cdots & \cdots & \cdots & \phi_{1 p}^{*} & 0 & a_{12} & \cdots & \cdots & a_{1 p} \\
\vdots & \ddots & & & \vdots & a_{21} & \ddots & \ddots & & \vdots \\
\vdots & & \ddots & & \vdots & \vdots & \ddots & \ddots & \ddots & \vdots \\
\vdots & & & \ddots & \vdots & \vdots & & \ddots & \ddots & a_{(p-1) p} \\
\phi_{p 1}^{*} & \cdots & \cdots & \cdots & \phi_{p p}^{*} & a_{p 1} & \cdots & \cdots & a_{p(p-1)} & 0
\end{array}\right)_{2 p \times 2 p}
$$

in which the upper left $p \times p$ and the upper right $p \times p$ matrix block coefficients are set to zero for lagged (exogenous) variables. That is, no variable observed at time directly predicts a variable at time- 1 , and variables at time- 1 cannot predict each other. The lower left and right blocks are $\boldsymbol{\Phi}^{*}$ and $\boldsymbol{A}$ matrices in Model 3.

The covariance matrix of the residuals in USEM is provided in the $\boldsymbol{\Psi}$ matrix:

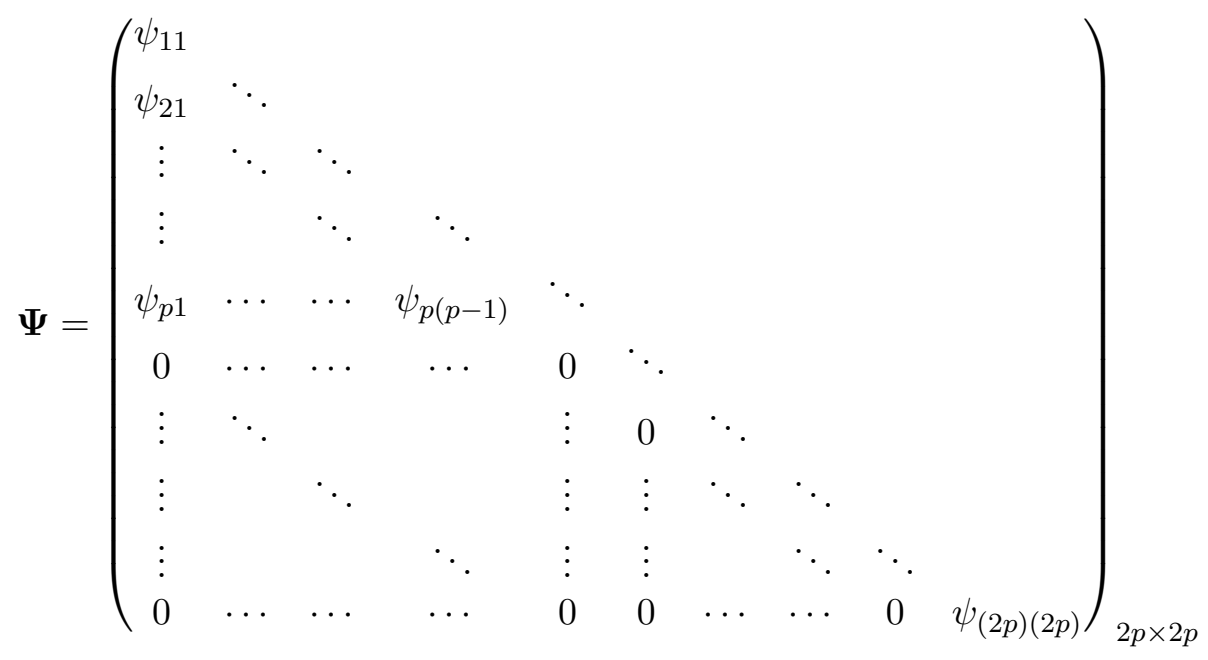

where the upper left triangle contains freely estimated variance and covariances among the lagged (exogeneous) variables, and the lower right triangle is the variance/covariance matrix of residuals as described in Model 3. Note that the covariances among residuals are set to zero in the traditional uSEM framework. These matrices provide the foundation from which the expansion to hybrid-uSEM is developed.

From uSEM to Hybrid uSEM

uSEM is extended into hybrid uSEM (huSEM) to represent a hybrid VAR by altering the residual covariance matrix $\boldsymbol{\Psi}$. Specifically, we relax the conditional independence 
assumption on the contemporaneous residuals of uSEM, and allow the errors be correlated:

$$
\zeta^{*} \sim N\left(\mathbf{0}, \Psi^{*}\right)
$$

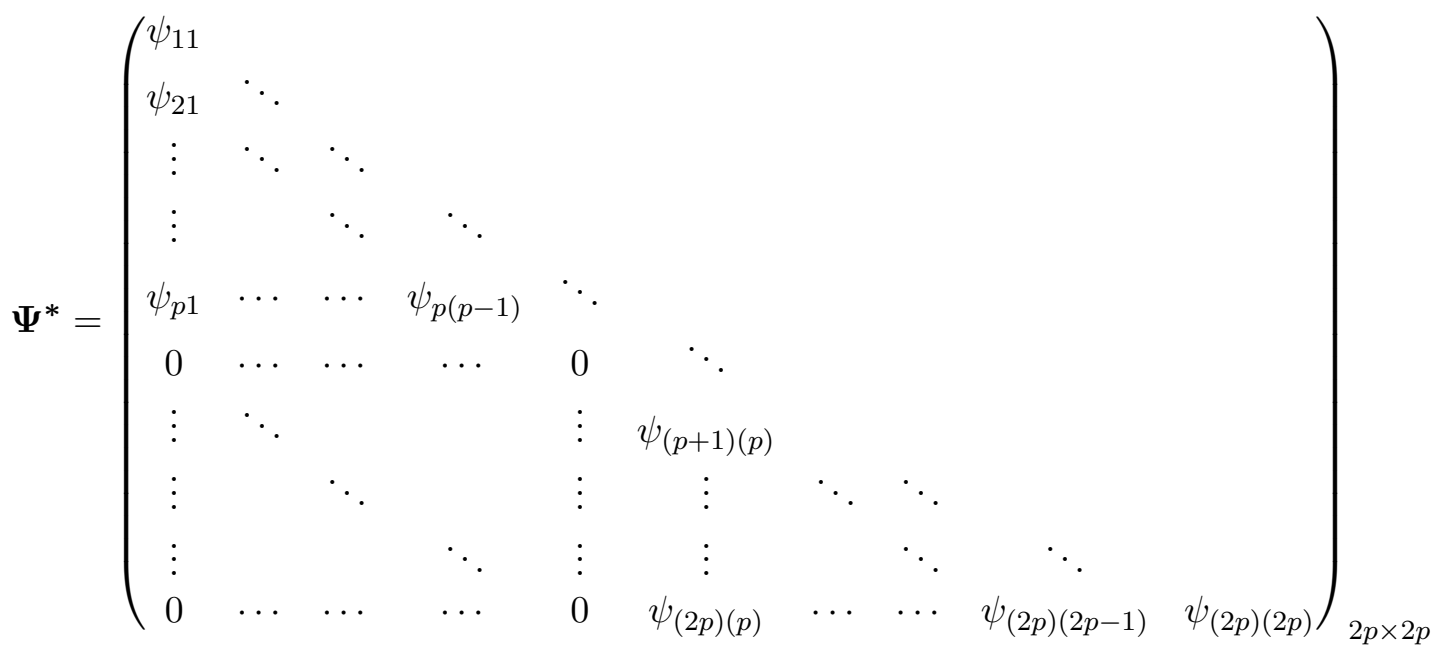

Note that the lower-right matrix block of $\boldsymbol{\Psi}^{*}$ is now a symmetric matrix with contemporaneous variances $\psi_{i}^{*}, i=p+1 \ldots 2 p$ of the residuals on the diagonal as seen in $\boldsymbol{\Psi}$. By substituting the off-diagonal 0's in $\boldsymbol{\Psi}$ with parameters of contemporaneous residual variance $\psi_{i j}^{*}, i, j=p+1 \ldots 2 p, i \neq j, \boldsymbol{\Psi}$ is turned to $\boldsymbol{\Psi}^{*}$. Each element in the lower right hand corner are now candidates in a model search procedure; we will not estimate all of them but provide them here for illustrative purposes. Additionally, the regression coefficient matrix $\boldsymbol{B}$ is turned to $\boldsymbol{B}^{*}$ as the relations and estimates may change from what they were previously, and the model-implied covariance matrix is now $\boldsymbol{\Sigma}^{*}$ derived by:

$$
\boldsymbol{\Sigma}^{*}=\left(\boldsymbol{I}-\boldsymbol{B}^{*}\right)^{-1} \boldsymbol{\Psi}^{*}\left(\boldsymbol{I}-\boldsymbol{B}^{*}\right)^{-1 \prime}
$$

In this hybrid VAR, the relation between two given variables $y_{t i}$ and $y_{t j}$ has the potential to be estimated by three parameters, ${ }_{i j}, a_{j i}^{*}$, and $\psi_{i j}^{*}$, with the first two representing a structure-type, directional relation, and the latter a covariance-type, bidirectional relation. In most cases they cannot exist simultaneously for identification purposes. If either $a_{i j} \neq 0$ or $a_{j i} \neq 0$, or both are true, then $\psi_{i j}=0$ should hold.

\section{A note on likelihood equivalence}

Likelihood equivalent models occur when models with two different structure and/or estimates result in the same likelihood value. There are two primary sources for models to be likelihood equivalent in the context of the time series models described herein. We describe both of them here as well as rationale for how model search approaches might still be able to identify the data generating models. The first source of model equivalence would occur when we wish to identify a relation between two variables. Let's consider variables $Y_{i}$ and $Y_{j}$ in this thought example. If the data were generated such that $Y_{i} \rightarrow Y_{j}$, one would have a hard time recovering this relation since $Y_{j} \rightarrow Y_{i}$ would provide an equivalent likelihood - and when standardized, and the same coefficient estimated. This standardized coefficient estimates would equal the correlation between the two variables. 
In the present modeling framework, this source of model equivalence is removed by the use of AR relations. Once a covariate is introduced this equivalence no longer holds.

Hence, $Y_{i}$ predicted by $Y_{j}$ as well as itself at a prior time point will not be likelihood-equivalent to $Y_{j}$ predicted by $Y_{i}$ and itself at the prior time point. In equation form, we can see that estimates would be the same for each equation if the variables are standardized:

$$
\begin{aligned}
y_{t, i} & =a_{i j} y_{t, j}+\zeta_{t, i} \\
y_{t, j} & =a_{j i} y_{t, i}+\zeta_{t, j},
\end{aligned}
$$

with $a_{i j}=a_{j i}$. Once the AR relations are included the coefficient estimates would differ:

$$
\begin{gathered}
y_{t, i}=a_{i j 1} y_{t, j}+\phi_{i i} y_{t-1, i}+\zeta_{t, i} \\
y_{t, j}=a_{j i 1} y_{t, i}+\phi_{j j} y_{t-1, j}+\zeta_{t, j} .
\end{gathered}
$$

Here, $a_{i j 1} \neq a_{j i 1}$. A similar finding results when examining the relations among residuals in that the presence of the AR relations enables for identification if whether coefficient $a_{j i 1}, a_{i j 1}$, or if the covariance of the residuals of $y_{i}$ and $y_{j}$ (after regressing out the AR effects) best portrays the contemporaneous relation among the two variables. They will no longer be likelihood equivalent. It is clear that the addition of the AR relations resolves this potential issue and underscores the importance of the Granger causality perspective.

The second source of equivalence is specific to the VAR, uSEM, and hybrid-uSEM models and requires more consideration. It has been shown previously that a VAR model with correlated residuals can be transformed to an equivalent uSEM model (Gates et al., 2010; Lütkepohl, 2005). It follows that hybrid-uSEMs similarly can also be transformed (Molenaar, 2017). While the transformations may be likelihood-equivalent, the coefficient estimates will not be identical. That is, the values obtained for, say, the correlation among residuals will not be the same value obtained for a standardized solution involving a contemporaneous value in the $A$ matrix for the same two variables. We provide an illustration of this in the Appendix A for interested readers. The reader is also referred to (Gates et al., 2010; Molenaar, 2017) for more extensive coverage of this problem.

The take home is that the coefficients will be altered if a VAR model is fit to data that is generated from a huSEM or uSEM model, and similarly, for a uSEM fit to either of the other models. When the coefficient values are higher in the original data generating model than in the transform, then search procedures should be able to detect the higher (true) path. Although, the data generating model may not always provide the higher coefficient - in that case, what may look like spurious results may arise, such as a contemporaneous directed path in the generative model being found in the covariance among residuals and/or the lagged $\Phi$ matrix when a VAR is fit. It is also possible that data generated to have a covariance among residuals may surface as a directed relation in the contemporaneous $\boldsymbol{A}$ matrix.

Given the equivalent (and thus accurate) models that emerge via transformation, when evaluating results from the VAR and USEM based approaches in our simulation study we focus mainly on detection of the presence of any contemporaneous relation between two variables. While we provide results on correct directionality recovery (i.e., directed contemporaneous, lagged, or bidirectional among residuals), this will be less of a focus due to the impossible task of true direction correct recovery for these models when data are generated 
under a huSEM framework. When evaluating huSEM results we consider the relation to be correctly recovered via presence for the same definition as VAR and uSEM and focus much more attention on the degree to which the approach recovered the relation in the data generating location.

\section{Model Searching: Forward-selection vs. Regularization}

To identify the hybrid model and estimate the covariance matrix defined by Equation 5 , some parameters need to be constrained as a saturated model (where $\boldsymbol{B}^{*}$ and $\boldsymbol{\Psi}^{*}$ are unconstrained) will be under-identified. Exploratory procedures in search for an identifiable model, often referred to as specification search (Kaplan, 1988; MacCallum, 1986) or modeling modification (Chou \& Huh, 2012), are used either when the theory to inform assumptions is lacking, or when identification or convergence issues exist due to any reason possible with time series data (e.g., insufficient data, high-dimensional variables, misspecifications) .

The exploratory search in SEM is often performed through a step-wise fashion using Lagrange multiplier score tests (Jöreskog \& Sörbom, 1986, modification indices MI). Built upon this, the current most widely-adopted specification search by uSEM is a forward-selection modeling building procedure proposed by Gates et al. (2010). This search begins with a null model, and the path with the highest and significant MI is added iteratively until the model arrives at an acceptable fit as indicated by two of four standard fit indices used in SEM: the NNFI (Bentler \& Bonett, 1980), CFI (Bentler, 1990), RMSEA (Steiger, 1990), and SRMR (Jöreskog \& Sörbom, 1981). Readers should refer to (Gates et al., 2010) for a detailed description or Gates and Molenaar (2012) for a large-scale simulation study identifying issues with this approach, namely the lower rates correctly detecting the true directionality of the contemporaneous relations in individual-level analyses. This individual-level model building procedure is available in the free open-source R package gimme (Lane, Gates, Fisher, et al., 2019), where the user simply provides the raw time series data and utilize the indSEM function.

In considering the optimal approach for arriving at sparse huSEM models, we opted against step-wise procedures for a variety of reasons. Extant literature has suggested that step-wise model build procedures in general are subjects to problems such as finding local solutions as a final model (thus missing an optimal model) and not being robust to misspecifications (Chou \& Bentler, 1990; MacCallum et al., 1992). These issues have been highlighted in the present context when conducting individual-level analysis since results may be driven by misspecifications made early in the search procedure (e.g., Gates \& Molenaar, 2012). Due to the discrete searching nature, results from forward selection depend heavily on the starting model, intermediate modification or stopping criteria, and are subject to local maxima. A more effective search for the optimal sparse model should be performed on a continuum (Huang et al., 2017). It is not to say that regularization can always distinguish likelihood equivalent models, but a global, continuous model search has a better chance to achieve the optimal model (closer to truth) over other possible likelihood-equivalent alternatives which include wrong parameters and misleading interpretations. By contrast, SEM estimated via maximum likelihood is limited in the number of variables it can handle, so a backward deletion approach that starts with a full model is not an option here. 
Regularization, on the other hand, will allow such a global search with all parameters undergo a continuous shrinking all at once. The term "regularization" (also referred to as "shrinkage" or "penalization") refers to the practice of imposing a penalty term to the cost functions controlling the size or the number of nonzero parameters. With a reasonable trade off of unbiasedness for estimation efficiency, regularization overcomes many modeling issues (e.g. overfitting, model complexity, under-identification), and have been widely used as variable selection and model estimation in contexts ranging from regression (Tibshirani, 1996), high-dimension reduction techniques (Zou, 2006), to graphical modeling (Friedman et al., 2008; for a broad overview, see Zou, 2006). Although historically less well received in SEM or psychometric modeling in general, recent developments of regularization method within the conventional SEM context (Huang, 2019; Huang et al., 2017; Jacobucci et al., 2019; Jacobucci et al., 2016) has opened up the possibility for adopting regularization to uSEM (Lane, 2017) and as an extension, huSEM.

Inspired by the advantage of using regularization in reaching a sparse graphical VAR model, the purpose of the present study is to develop a similarly flexible modeling and estimation approach for hybrid uSEM (huSEM). This is necessary because for one, traditional VAR cannot offer a holistic picture of possible contemporaneous relations no matter how efficient the model search algorithm is since it does not include the possibility of directed contemporaneous relations. Two, while uSEM models allow for structural type of contemporaneous relations among observed variables, it does not allow for contemporaneous covariances among the errors and typically model searches are conducted without regularization. Below we first demonstrate the extension of uSEM to regularized uSEM (i.e., Reg-uSEM) and hybrid VAR to regularized huSEM (i.e., reg-huSEM), following the framework of regularized SEM (Jacobucci et al., 2016). In our simulation study, we evaluate if the proposed global-searching Reg-uSEM and Reg-huSEM are indeed superior to the modification index-based stepwise USEM and huSEM search procedures, respectively. Our prediction is that Reg-huSEM will surface as the optimal model in terms of recovering the data generating relations. Our intention is to implement it under the context of huSEM not only serves to facilitate the applicability of more flexible VAR models for intraindividual variability research in practice, we also view this a new step forward for regularized SEM under time series context.

\section{Model Expression and Estimation for A Sparse hybrid VAR: Reg-huSEM}

The Reg-SEM approach uses regularized maximum likelihood estimation (MLE) to derive an implied covariance matrix imported to the statistical program. When fitting a Reg-huSEM, a penalty function is added to the MLE cost function of uSEM, $F_{M L}(\theta)$ :

$$
F_{R e g}(\theta)=F_{M L}(\theta)+\lambda P\left(\theta^{*}\right)
$$

Where

$$
F_{M L}(\theta)=\ln \left|\boldsymbol{\Sigma}^{*}\right|+\operatorname{tr}\left[\boldsymbol{S} \boldsymbol{\Sigma}^{*-1}\right]-\ln |\boldsymbol{S}|-p
$$

Where the model implied covariance $\Sigma^{*}$ is given by Equation 5 . Note that substitute $\Sigma^{*}$ by $\Sigma$ will reduce it to a Reg-uSEM. $S$ is the observed covariance matrix, $p$ is the number of model parameters. The penalty function $P\left(\theta^{*}\right)$ is the general function (see below for details) to sum up the values of all the regularized parameters by a user-defined penalty method, with the 
level of regularization or sparsity controlled by a tuning parameter $\lambda$. $\lambda$ controls the amount of shrinkage such that when $\lambda$ equals zero, Equation 8 reduces to MLE; as $\lambda$ increases to infinity, the penalized parameters are shrunken towards zero.

To estimate a Reg-uSEM or Reg-huSEM using the regsem function, parameters in $P\left(\theta^{*}\right)$ include all the regression coefficients, i.e., $\boldsymbol{\Phi}^{*}$ and $\boldsymbol{A}$ matrices of the $\boldsymbol{B}^{*}$ matrix, as well as covariance of contemporaneous variables, $\psi_{i j}, i, j=p+1, \ldots, 2 p, i \neq j$ elements in $\Psi^{*}$ for huSEM, with the level of penalty being controlled by $\lambda$. The model does not penalize the variance parameters or covariance of lagged variables $\psi_{i j} i, j=1, \ldots p, i \neq j$. Our particular interest is the shrinkage of the three parameters: a) $a_{i j}$; b) $a_{j i}$; and c) $\psi_{i j}$, that estimate contemporaneous relation between variables $Y_{i}$ and $Y_{j}$. Ideally, the optimal of $\lambda$ (with the lowest BIC) penalizes all unnecessary parameter(s) to zero and estimate the remaining parameter(s), unraveling the true type of relation from a), b), or c). In comparison, only regression weight parameters in $\boldsymbol{B}$ will be penalized when fitting Reg-uSEM, as there are not any free parameters for the covariances of contemporaneous variables for the contemporaneous relations between variables $Y_{i}$ and $Y_{j}$. If neither, only one, or both of $a_{i j}$ and $a_{j i}$ remain nonzero under optimal $\lambda$, they present null, a unidirectional, or a reciprocal contemporaneous relation between $y_{i}$ and $y_{j}$, respectively.

The two most common basic regularization methods are the $L 2$-norm (i.e., ridge; Hoerl \& Kennard, 1970), which penalizes the sum of the squared values of the parameters, and the L1-norm (aka. the least absolute shrinkage and selection operator, LASSO; Tibshirani, 1996), which penalizes the sum of the absolute values of the parameters. There are also various alternative forms and adaption of the two has been developing vastly, for instance, elastic net, SCAD, and MCP are widely used in practice (Hastie et al., 2015; Zou \& Hastie, n.d.).

Penalized parameters are shrunken as $\lambda$ increases, but they can reach exactly zero only under L1-penalty. Hence, LASSO penalty is often used in favor of variable selection, while ridge penalty is chosen when minimizing predictor error is a priority. LASSO estimation introduces sparsity by imposing the penalty, the level of which is gauged by searching across a pre-specified range of $\lambda$ values until the optimal $\lambda$ (hence the sparsity level) is reached such that the model has the least mean square error (i.e., the lowest BIC).

Because of our goal to reach a sparse model in order for huSEM (and in the process, uSEM) to have a unique solution, we examine the use of two LASSO-types of penalty: the LASSO and adaptive LASSO (aLASSO; Zou, 2006). That is, L1-norm is the functional form for $P(\theta)$. The idea is that when one parameter is shrunk to zero, the model gains one degree of freedom. We need LASSO-penalty to identify the true zero parameters for a sparser model. It has been shown that the number of nonzero coefficients is an unbiased estimate of the degrees of freedom for the model (Zou, 2006). As the penalty increases and more selected parameters are set to zero, the degree of freedom increases and hence goodness of fit. Although the gained degree of freedom can not ensure a unique solution for the remaining (true nonzero)

parameters, it eliminates the possibility of under-identification resulting from over-parameterization, and thus increases the chance of model identification and model fit. To balance the introduced bias, the aLASSO was developed so as to adjust for bias in large coefficients by imposing parameter-specific weighted $L 1$-penalty. In aLASSO, each regularized parameter is scaled by the corresponding free MLE estimate such that large coefficients are less penalized. Both penalty methods are implemented in the current version of $\mathrm{R}$ package regsem (Jacobucci et al., 2016). An added benefit of regsem program is that it inherits model 
specification and syntax language from the most popular R program for SEM, lavaan (Rosseel, 2012).

A popular optimization method for penalized likelihood estimation is proximal gradient descent, a two-step procedure in each iteration, where step-one involves a method for calculating the step size and in step-two a soft-thresholding operator is used to overcome non-differentiability of the LASSO penalty at the origin (for more technical details on optimization, see Hastie et al., 2015; Jacobucci, 2017). Similarly, the package graphicalVAR employs a hybrid LASSO type of regularization to jointly estimate the temporal and contemporaneous network structures (Abegaz \& Wit, 2013), extending upon the multivariate regression with the covariance estimation (MRCE) algorithm (Rothman et al., 2010). The joint regularization method involves iteratively optimizing edges of the lag-1 PDC (i.e., the temporal network) by searching across a range of $\lambda_{1}$ ), using cyclical-coordinate descent, and the lag-0 PCC (i.e., the contemporaneous network) by $\lambda_{2}$ ), using the graphic LASSO algorithm (Friedman et al., 2019), until model converges with the optimal pair of $\left(\lambda_{1}, \lambda_{2}\right)$ that gives the least mean square error loss indicated by the lowest extended BIC.

\section{THE SIMULATION STUDY}

The simulation study aims to serve two major goals: 1) to validate whether the huSEM can correctly recover the data generating relations when directed and undirected contemporaneous relations are both presented in addition to AR and cross-lagged effects among multivariate time series, regardless of the model search procedures. At the same time, we will assess to what extent the other VAR variations (i.e., uSEM and gVAR) can recover the presence of relations even though the data generation does not match the modeling approach; and 2) to evaluate whether regularization performs better than does forward selection for the uSEM and huSEM models. We test if model recovery performance differs between the adaptive LASSO and the LASSO penalty when applied to uSEM or to huSEM, respectively. Regarding the stepwise model search, emerging work suggests the stepwise approach performs well when conducted within the GIMME algorithm (Luo et al., Under Review). However, it remains unknown how it will perform within the individual-level search described above. In proposing the utilization of regularization framework, we thus additionally evaluate huSEM using the stepwise MI approach, which we term here MI-huSEM to distinguish it from the Reg-huSEM (similarlty for MI-uSEM and Reg-uSEM). Related, we are interested in assessing the consequence of model misspecification for contemporaneous relations in different limited VAR models (i.e., undirected contemporaneous relations in uSEM models and directed in gVAR models). We evaluate these modeling framework with the consideration of the impact from conditions including the amount of variables, strength of relations, as well as number of timepoints.

Data were generated using the hybrid VAR representation that is flexible enough to allow for both types of contemporaneous relations. Following a transformation of Equation 3 (Gates et al., 2010, see Appendix A, supplemental code, and), directional contemporaneous and lagged relations (i.e., autoregressive and cross-lagged) were incorporated as regression coefficients in $\boldsymbol{B}^{*}$, while bidirectional contemporaneous connections were incorporated as marginal residual covariances in $\mathbf{\Psi}^{*}$. We prespecified a stationary model that included a mix of different relations and chains of connections (see Figure 1). For example, in the 10-variable 
FiguRE 1

Data Generating Model (the 5-variable and the 10-variable)
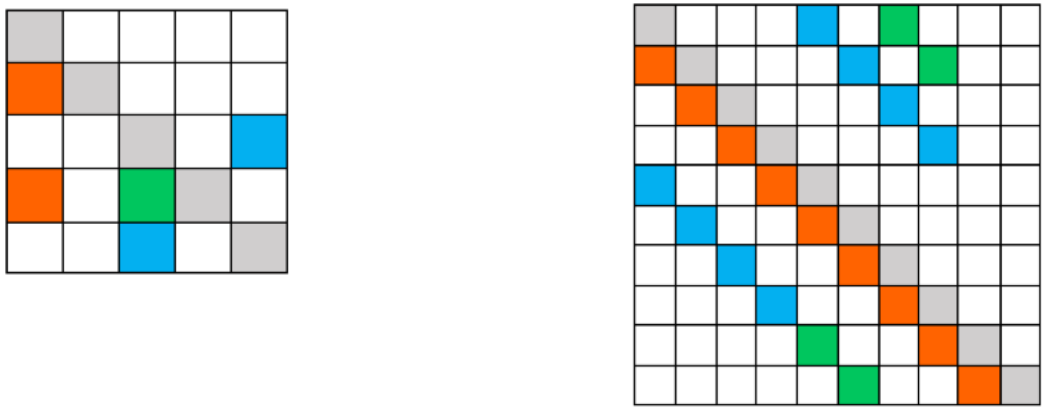

Notes: Orange - contemporaneous regression coefficients where $a_{i j} \neq 0$ or $a_{j i} \neq 0$, and $\psi_{i j}=0$; Green - Crosslagged regression coefficients where $\phi_{i j} \neq 0$ or $\phi_{j i} \neq 0$, and $\psi_{i j}=0$; Blue - contemporaneous covariance coefficients where $\psi_{i j} \neq 0, a_{i j}=0$ and $a_{j i}=0$.

model, each variable has a directed contemporaneous relation with a neighbor variable; variable 7 has a lagged effect on variable 1 , and variables 1 and 5 have contemporaneous covariances.

\section{Simulation Design Conditions}

We designed the simulation to evaluate the models of interest by varying the number of timepoints, number of nodes, and path strength for each of the types of conditions. Our simulations were fully crossed. The choice of these design factors was decided such that they represent data structure and characteristics of time series data in psychological and psychophysiological research.

\section{Number of timepoints}

We varied the number of time points across three levels: $T=60,200$, and 1000, representing a range of potential time lengths encountered in practice. A previous simulation study had validated the use of MI-uSEM with as few as T $=60$ time points (Lane, Gates, Pike, et al., 2019), which is also close to the sample size common in daily diary studies (e.g., Wright et al., 2015). Time series data derived from neuroimaging or psychophysiology studies (e.g. fMRI, EEG) typically range from 200 to 1200 time points (Smith et al., 2011). We chose 200 and 1000 to represent this range.

\section{Number of variables}

We varied the number of variables in two levels: a 5-variable model and a 10-variable model. This choice corresponds to previous simulation studies emulating time series data obtained from brain studies (Smith et al., 2011). We were interested in finding to what extent the increased complexity of a larger model (more connections per variable) influences the 
performance of path recovery and/or the elimination of false paths. For simplicity, we obtained a moderately dense model with roughly $10 \%$ path density for both the smaller and larger models. This leads to roughly one relation per variable (as the outcome) for the 5-variable model and on average two relation per variable for the 10 -variable model. The choice of a relatively moderate number of variables was made for the sake of computational feasibility. Because current regularization algorithm is limited both in the computation speed and the number of regularized variables in the model.

\section{Strength of relationship}

We evaluated the extent to which the path and direction recoveries are a function of the strength of the relation. Recall that the focus of our investigation is the identification of true connections that are a mix of cross-lag, directed contemporaneous, and bidirectional contemporaneous relations. To avoid confounding effect between path type and path strength, we altered path strength by path type in the way such that in each condition paths falling to one of the two strength categories: moderate and strong ${ }^{1}$. For instance, in one condition all of the directed contemporaneous relations are considered strong with a uniform magnitude while those of the other two types of relations were relatively moderate (also with a uniform magnitude). As for the magnitude of path strength, previous simulation had used a mean of .40 and a standard deviation of .1 , with a range limited to $(.20, .60)$. To keep it close to these prior studies and simple, we used .7 for strong relations, and .25 for the moderate relations in the 5 -variable model. Since each variable has more relations in the 10 -variable condition, the strengths had to be decreased to prevent instability of the data. Here, .45 for strong relations, and .25 for moderate ones for the 10-variable model. This helped to avoid inflated variances in the endogenous variables for the larger sized model. In addition, all variables in the DGMs for either number of variables were generated to have what we consider a moderate autoregressive effect (.25). This was to mimic a typical empirical scenario where some lagged relations are expected. Note that this is a weaker AR effect size than is often used in simulation studies (Epskamp et al., 2018; Gates et al., 2017).We chose this value as it may impact the accurate recovery of directions in the step-wise approach (Lane, Gates, Pike, et al., 2019; Weigard et al., Under Review) yet it was unclear the impact on regularized methods.

\section{Analytic Procedures}

All datasets are generated, modeled, and analyzed in R (R Core Team, 2019). For the goals described before, each dataset was fitted by a combination of three model representations (i.e., uSEM, hybrid-uSEM, VAR) mixed with three types of modeling framework (i.e., MI with forward selection, regularization under SEM, and regularization under GGM). Specifically, uSEM and huSEM are evaluated by MI and regularized SEM with two penalty methods (LASSO and aLASSO). VAR (with covariances allowed among residuals) is modeled by GGM with the regularization method described above. This leads to seven models under investigation: MI-uSEM, Reg-uSEM with LASSO, Reg-uSEM with aLASSO, MI-huSEM, Reg-huSEM with LASSO, Reg-huSEM with aLASSO, and gVAR with MRCE regularization

\footnotetext{
${ }^{1}$ Note that these are arbitrary and relative terminology used for ease in conveying the results.
} 
(see Table 1). Data fitted by MI-uSEM and MI-huSEM used the indSEM function under (Lane, Gates, Fisher, et al., 2019) package. The latter used the recently introduced huSEM stepwise approach (Luo et al., Under Review) available as an option in the gimme package (Lane, Gates, Fisher, et al., 2019). We regularized gVAR using graphicalVAR (Epskamp, 2018). We adopted a fully-crossed simulation design with the aforementioned factors, resulting in $2 \times 3 \times 3=18$ data generating conditions, each with 1,000 datasets.

Reg-uSEMs and Reg-huSEMs were conducted using the cv_regsem function in the regsem package (Jacobucci et al., 2019). The following major three-step procedure is needed in fitting a Reg-uSEM or Reg-huSEM: 1) specify and estimate a uSEM or huSEM model in lavaan, where the model implied covariance $\Sigma$ is imported to a lavaan function, and an (unpenalized) MLE cost function $F_{M L}(\theta)$ is optimized; 2) the lavvan object from step 1) is input to regsem function and the model structure matrices are transformed to the Reticular Action Model notation (RAM; McArdle \& McDonald, 1984, McArdle, 2005); 3) perform regularized estimation on the cost function $F_{\text {regsem }}(\theta)$, with user-specified penalty method and selected parameters in $P\left(\theta^{*}\right)$.

For example, in our DGM where $p=5$ or $p=10$,

$$
P\left(\theta^{*}\right)=\left\|\phi_{i j}, \beta_{k l}, \psi_{m n}\right\|_{l 1}
$$

in which

$$
\begin{gathered}
i, j=1, \ldots, p, i \neq j ; \\
k, l=1, \ldots, p, k \neq l \\
m, n=p+1, \ldots, 2 p, m \neq n
\end{gathered}
$$

For models that converged, results of the optimal model with the smallest BIC are returned. Note that in the circumstance of an under-identified model, results from the optimization of $F_{M L}(\theta)$ might be untrustworthy, but lavaan (used within the package gimme) will return MLE estimates regardless. This is also the MLE weights in aLASSO, which is a downside of using parameter-specific penalty method when the unpenalized model fails to

\begin{tabular}{|c|c|c|c|c|c|c|c|}
\hline Models & $\begin{array}{l}\text { Model 1: } \\
\text { gVAR }\end{array}$ & $\begin{array}{l}\text { Model 2: } \\
\text { MI-uSEM }\end{array}$ & $\begin{array}{l}\text { Model 3: } \\
\text { RL-uSEM }\end{array}$ & $\begin{array}{l}\text { Model 4: } \\
\text { RA-uSEM }\end{array}$ & $\begin{array}{l}\text { Model 5: } \\
\text { MI-huSEM }\end{array}$ & $\begin{array}{l}\text { Model 6: } \\
\text { RL-huSEM }\end{array}$ & $\begin{array}{l}\text { Model 7: } \\
\text { RA-huSEM }\end{array}$ \\
\hline $\begin{array}{l}\text { lag-0 } \\
\text { (Directed/Undirected) }\end{array}$ & $\mathrm{U}$ & $\mathrm{D}$ & $\mathrm{D}$ & $\mathrm{D}$ & $\mathrm{D} / \mathrm{U}$ & $\mathrm{D} / \mathrm{U}$ & $\mathrm{D} / \mathrm{U}$ \\
\hline $\begin{array}{l}\text { Est.Method } \\
\text { (Regularization/pseudo MLE) }\end{array}$ & Reg & pMLE & Reg & $\operatorname{Reg}$ & pMLE & Reg & Reg \\
\hline R Package & graphicalVAR & gimme & $\begin{array}{l}\text { lavaan, } \\
\text { regsem }\end{array}$ & $\begin{array}{l}\text { lavaan, } \\
\text { regsem }\end{array}$ & gimme & $\begin{array}{l}\text { lavaan, } \\
\text { regsem }\end{array}$ & $\begin{array}{l}\text { lavaan, } \\
\text { regsem }\end{array}$ \\
\hline Search Algorithm & MRCE & Forward & LASSO & aLASSO & Forward & LASSO & aLASSO \\
\hline Missing Data Allowed & No & Yes & No & No & Yes & No & No \\
\hline
\end{tabular}
achieve identification.

TABLE 1.

Models and Estimation Approaches

Notes: aLASSO = adaptive LASSO. Model 2-4 or Model 5-7 represent the same VAR models, they only differ in model search and estimation approaches, respectively. Model names in red indicate the data generating model. 


\section{Outcome Measures}

Because our primary goal is to compare model performance in terms of reliably recovering relations among variables with a specific direction, we used sensitivity and specificity measures for the evaluation. Sensitivity and specificity are both popular outcome measures for evaluating capability of recovery of connections in network research (e.g., Abegaz \& Wit, 2013; Epskamp \& Fried, 2016). Sensitivity is calculated by the ratio of the true positive count discovered in the search over the sum of all true relations in the data-generating model (i.e., true positives and false negatives). Sensitivity represents the power to detect true relationships. Specificity, in comparison, is calculated by the ratio of true negative count over the sum of negatives in the DGM (i.e., the sum of true negative count and false positive count). This represents the percentage of non-existing paths in the data generating model that the search procedure accurately omitted in the final model. These measures allow for a global evaluation of a model's ability to detect true recovery and to reject false ones. In both sensitivity and specificity measures, higher values indicate better performance in terms of the selection of true data-generating relations. We do not assess bias or variance in parameter estimation because models different from data generating model are fitted to the data. Due to misspecification in some part of the fitted models (except the hybrid-uSEM), models have different sets of possible parameters from the DGM. The values of which are thus not comparable and the concept of bias is useful here.

Importantly, we examined sensitivity and specificity for lagged, regression and covariance contemporaneous relationships separately. Additionally, towards the special interest for the direction of contemporaneous relation, we distinguish presence sensitivity from direction sensitivity. Presence sensitivity refers to the identification for the existence of a relation between two variables regardless of the direction associated with such relation (including $X \rightarrow Y, X \leftarrow Y, X \leftrightarrow Y$, or $X-Y$ ). Direction sensitivity refers to the more precise recovery of not only the existence but also the correct direction ( $X \rightarrow Y$ would be distinguished from $X \leftarrow Y$ for directional contemporaneous, bidirectional can be covariance in the uSEM model $X \leftrightarrow Y$ or partial correlation in the gVAR model $X-Y$ ).

Since it has been shown above that uSEMs can be transformed to equivalent VAR models with correlated residuals, it is critical to note that our method of assessing presence separately from direction accommodates this. We also note that presence sensitivity or direction sensitivity might suggest different forms of representation across models, due to the fact that the models differ in how contemporaneous relations are specified. For instance, since contemporaneous effects are only specified as undirected (i.e., partial correlation) in the gVAR modeling framework, the presence recovery of a true directed contemporaneous relation (e.g., $V 1 \rightarrow V 2$ in the DGM Figure 2) refers to the recovery of undirected contemporaneous relation (i.e., $V 1-V 2$ in the PCC of Figure 3). Epskamp et al. (2018) and Gates et al. (2010) both illustrated that the directed contemporaneous relations surface as an undirected contemporaneous edge in PCC plus an additional lagged edge in PDC, as correspond to the directed contemporaneous structure in the SVAR model (e.g., $V 1 \rightarrow V 4$ in the PDC of Figure $3)$. For this reason, we would count these lagged relations as correct presence and direction recovery (for the true path $V 1 \rightarrow V 4$ in Figure 2). Similarly, for uSEM models any covariance among the residuals (e.g., $V 3 \leftrightarrow V 5$ in Figure 2 will be forced into contemporaneous directed paths with an arbitrary direction (e.g., $V 3 \rightarrow V 5$ in Figure 4), and the correct "direction" of 
Figure 2.

Data Generating Model: the 5-variable huSEM

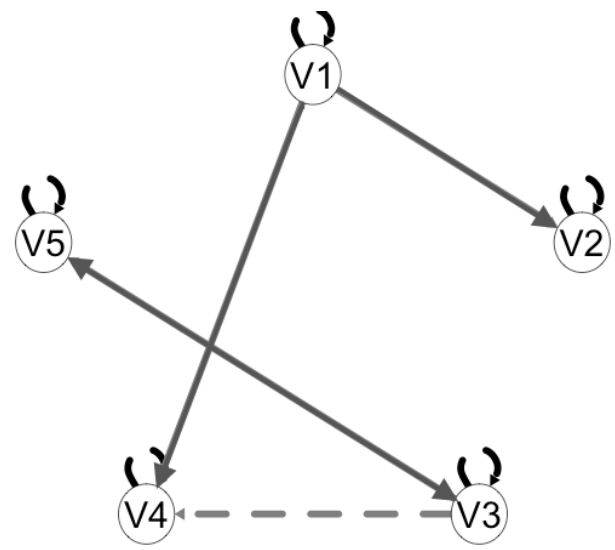

Figure 3.

The 5 -variable huSEM recovered by a gVAR model

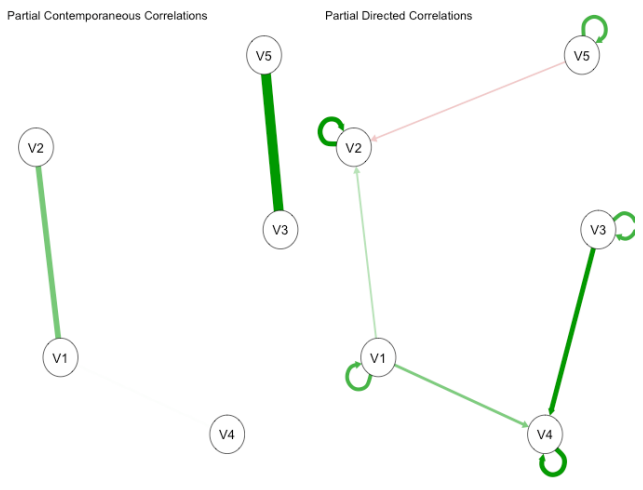

FiguRE 4.

The 5 -variable huSEM recovered by a uSEM model

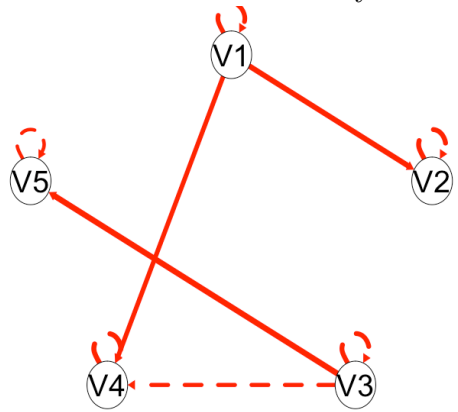

Note: Plots are consistent with those provided by the respective packages. For the uSEM results from gimme, red indicates positive estimates and blue for negative. Solid lines represent lag- 0 paths and dashed lines indicate lag-1 paths. For gVAR results from graphicalVAR, green lines indicating positive edge and red for negative. Line width correspond with coefficient estimate size for both.

bidirectional would not have been found (Gates et al., 2010). Contemporaneous covariance recovered as directed paths are thus considered as presence (but not direction) recovery. 
However, in uSEMs the recovery of the presence of a contemporaneous path includes regression coefficients of either direction (i.e., $V 1 \rightarrow V 2$, or $V 1 \leftarrow V 2$ ), and in huSEMs the regression coefficients and residual covariances (i.e., $V 1 \rightarrow V 2, V 1 \leftarrow V 2$, or $V 1 \leftrightarrow V 2$ ). The direction recovery is the rate of the true path only, i.e., $V 1 \rightarrow V 2$. Similarly, for the recovery of a data generating bi-directional marginal contemporaneous correlation $(V 3 \leftrightarrow V 5)$, a relation not incorporated in uSEMs and as partial correlation in gVAR, the presence recovery include contemporaneous $V 3 \rightarrow V 5$ or $V 3 \leftarrow V 5$ for the uSEM and $V 3-V 5$ for the gVAR. The corresponding direction recovery rate is zero if only one of the two directed path was recovered in uSEM, while it equals to that of the presence recovery in gVAR.

\section{RESULTS}

As a reminder, the huSEMs discovered with MI, LASSO, or aLASSO regularization were the only models where all forms of paths were allowed in the model search space. Hence these are the only onees we expect to perform well both in terms of recovering the presence of a relation as well as the direction type. The MI-uSEM and reg-uSEM models have the same model structure as each other; they differ only in model estimation method. The gVAR model also has a different structure from huSEMs, and is estimated under GGM framework using regularization. Per our interest in the recovery of different types of relations, below we present path recovery and direction recovery for each form across the modeling approaches, respectively. Note that there are some cases where the models in $\mathrm{cv}_{-}$regsem did not converge, especially for the reg-uSEM when fitted to data with 10 variables and a small to moderate sample size (see Appendix B, Table 2 for the details). For the sensitivity and specificity results, the counts were calculated using all the converged models. Model nonconvergence is a complex issue, which we will return to in the limitation section.

\section{Sensitivity for Directed Contemporaneous Relations}

$g V A R$

Recovery rates for gVAR model are indicated by the blue dashed lines in Figures 5 to 9. Since directed contemporaneous relations cannot be specified according to the DGM, the presence recovery rate of contemporaneous associations was calculated as the percent of undirected edges in the lag-0 PCC contemporaneous network and directed edges in the PDC lagged network across all true paths (Figure 5). As expected from the transformation of hu-SEM to VAR, the presence of directional contemporaneous relations introduced a directed edge between corresponding nodes in the lag-1 PDC network, this recovery was consistently found when such structural path was strong and the sample size was large. This confirmed that the presence and direction of a directed contemporaneous relation were recovered separately by two edges in gVAR, resulting in a less parsimonious model. While the undirected edge in lag-0 PCC only reveals the presence, the direction information was disclosed by a temporal sequential effect in lag-1 PDC. However, the performance of the direction recovery (Figure 6) was less consistent, varying across sample sizes and the relative strength of the relations. For a strong directed contemporaneous relation, the presence was nearly always recovered when $\mathrm{N}=$ 1,000 (left column in Figure 5), and direction was correctly disclosed by the lag-1 relation (see 
FiguRE 5 .

Path Presence Sensitivity (Y-axis) for Directed Contemporaneous Relation by Timepoint (X-axis), Strength (column), and Dimension (row)

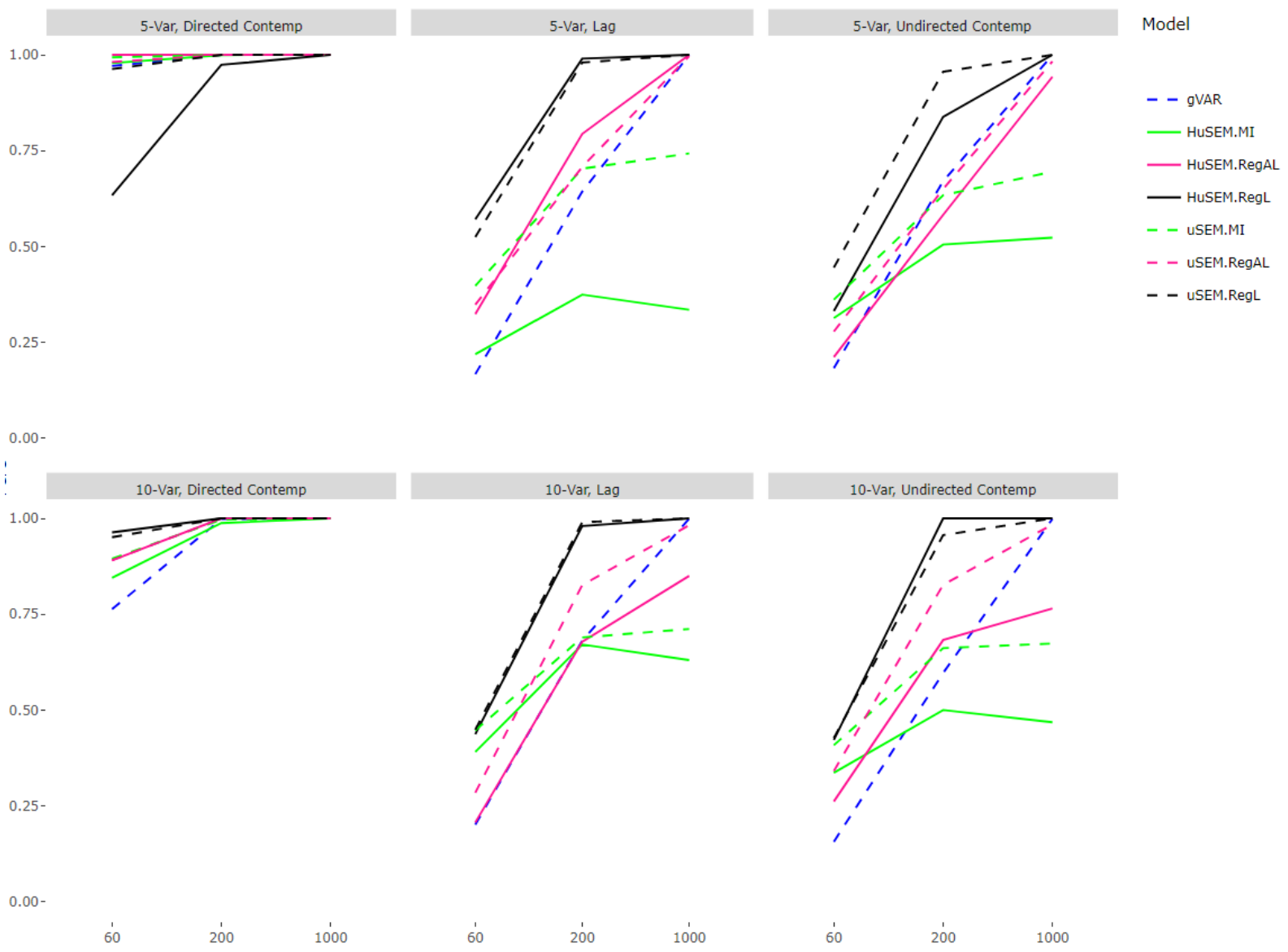

Note: 5 -Var $=5$-variable model, 10 -Var = 10-variable model; "V5-Var, Directed Contemp" means the DGM is 5 -variable model and the strongest type of relation is the directed contemporanous; x-axis represents number of timepoint (from the lowest on the left to the highest on the right); y-axis represent the $\%$ of the recovery; models are line coded in such a way that solid lines represent correctly specified models while dashed lines for alternative representations, and model search procedures are color coded.

left column in Figure 6). When such relations were moderate, the presence (middle and right columns in Figure 5) was still recovered given a sufficient sample size $(100 \%$ for $\mathrm{N}=1,000$, $60-70 \%$ for $\mathrm{N}=200$, and $16-20 \%$ for $\mathrm{N}=60$ ), but the direction (middle and right columns in Figure 6) was no longer recovered consistently as cross-lagged relation (50\% with $\mathrm{N}=1000$, and $10 \%$ for $\mathrm{N}=60$ or 200 ). The tendency of gVAR to recover one directed contemporaneous connection by two edges (i.e., one being undirected and one being lagged) might very likely decrease the power to recover other true connections in the entire model and increase the chances of recovering false positive edges that provide more parsimonious models. 
Figure 6 .

Path Direction Sensitivity (Y-axis) for Directed Contemporaneous Relation by Timepoint (X-axis), Strength (column), and Dimension (row)

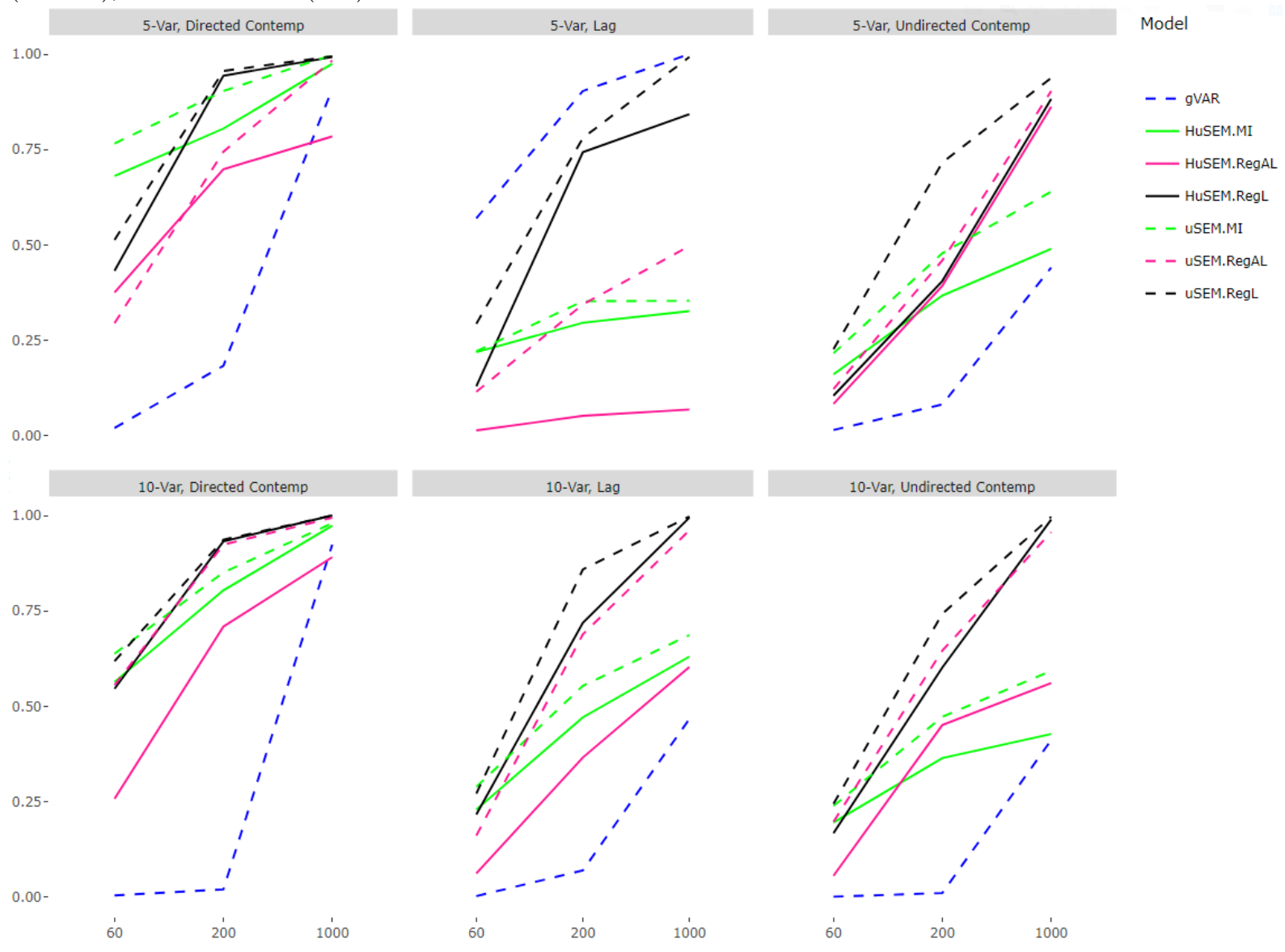

MI-uSEM and Reg-uSEM (with LASSO or aLASSO penalty)

We similarly found that all uSEM approaches showed excellent presence sensitivity for strong directed contemporaneous relations among converged models, with recovery being highest for the MI approach for the 5 variable condition across the time length conditions. When the directed contemporaneous paths were moderate, the Reg-uSEM models (black and pink dashed lines) outperformed MI-uSEM (green dashed line) in recovering relations with moderate strength (middle and right columns) but did not perform as well as gVAR when the lagged relations were strong. The presence recovery of a strong path was excellent for MI-uSEM, and the direction recovery was near perfect ( $100 \%$ for $\mathrm{V}=5$ and $98 \%$ for $\mathrm{V}=10$ ) when $\mathrm{T}=1000$, with these values slightly decreasing for presence recovery as $T$ decreased. Recovery of the true directionality was barely influenced by the decreasing number of time points for the strong condition. In comparison, the presence recovery of a moderate contemporaneous path was not satisfactory across conditions. In terms of Reg-uSEMs, when $T \geq 200$ both Reg-uSEM estimation approaches exhibited perfect presence sensitivity for the directed contemporaneous relation when that relation was strong and $T=1000$. Among the two penalty methods, LASSO (black dashed line) outperformed aLASSO (pink dashed line) in 
both types of recovery. For example, with a medium sample size, the presence recovery rate were $96-99 \%$ for LASSO-uSEM, compared with 65-83\% for aLASSO-uSEM. It was found that direction recovery depended largely on sample size, despite the strength of the relation, with poor recovery occurring for the $T=60$ condition. For example, for a small-size small-sample model, nearly half of the presence recovery by LASSO was associated with an opposite direction, this was even worse for aLASSO. The false direction issue was immediately improved with an increasing sample size: for LASSO, it dropped to about one fourth with a medium sample size and almost eliminated when sample size hit 1000. It is surprising that even with a medium to large sample size, about $30 \%-50 \%$ of the presence recovery by aLASSO was associated with a wrong direction. Interestingly, direction recoveries were slightly higher overall in the 10-variable model than that of the 5-variable model overall (e.g., in the 10-variable model, false direction only emerged when sample size was small: $30-40 \%$ for $\mathrm{T}=$ 60 , and $13-22 \%$ for $\mathrm{T}=200$ ). In summary, when the relation is strong, MI-uSEM has higher detection of contemporaneous relations and specificity of the direction. For smaller coefficient values, Reg-uSEM with LASSO penalty was the best choice.

\section{MI-huSEM and Reg-huSEM (with LASSO or aLASSO penalty)}

Results of Reg-huSEM using LASSO penalty (black solid line) was similar to that of Reg-uSEM using LASSO penalty (black dashed line), while the other two models actually underperformed their uSEM counterparts (note that directed contemporaneous relations are correctly specified in all uSEM models). The recovery rate of MI-huSEM (green solid line) for moderate directed relations was particular low, even worse than those of MI-uSEM (green dashed line). For Reg-huSEM with aLASSO penalty (pink solid line), although the presence recovery was similar to those of its uSEM counterpart (pink dashed line), the direction recovery dropped substantially. This resulted from even more cases of presence recovery with a wrong direction for a structural contemporaneous connection. Reg-huSEM with aLASSO penalty was at the highest risk of committing this false positive, and it was more likely to occur with small-to-medium sample sizes. While these only counted for a small portion, this explained part of the bigger gap between the presence and direction sensitivity among huSEMs compared to that of uSEMs.

Taken together, we found that while all models can reliably recover the presence of a strong structural contemporaneous relation, only Reg-uSEM and Reg-huSEM with LASSO penalty can accurately recover the presence of a moderate relation along with the direction of such relation. We note that This might be an overly optimistic result for Reg-uSEM though recall that this result must be considered in light of the fact that the result is based on the converged models while in fact a certain portion of the 10-variable Reg-uSEM models in some condition failed to reach convergence. Interestingly, the aLASSO penalty method has the tendency to introduce directed paths with a false direction, no matter in Reg-uSEM or Reg-huSEM. In comparison, the stepwise approach typically only performed well for paths that had strong coefficient sizes. MI-uSEM is not sensitive to a moderate relation. This is likely due to the algorithm favoring parsimony, and stopping the search after the strong relations were recovered and variability in the variables adequately explained. The gVAR performed well in terms of presence recovery for the moderate contemporaneous relations and did not experience convergence issues for the 5-variable condition. The gVAR approach 
typically did not recover directed contemporaneous relations very frequently when $T<1000$ and in the 10 variable condition. When sample size is sufficiently large $(T=1,000)$, presence of directed contemporaneous paths were estimated as undirected edges in PCC (i.e., partial correlation), comparable to the bidirectional covariance in the USEM or huSEM models. The direction is recovered as the corresponding lagged effect only under favorable condition and when this relation is sufficiently strong.

\section{Sensitivity for Undirected or Bi-directional Contemporaneous Relation}

FiguRE 7.

Path Presence Sensitivity (Y-axis) for Undirected Contemporaneous Relation by Timepoint (X-axis), Strength (column), and Dimension (row)

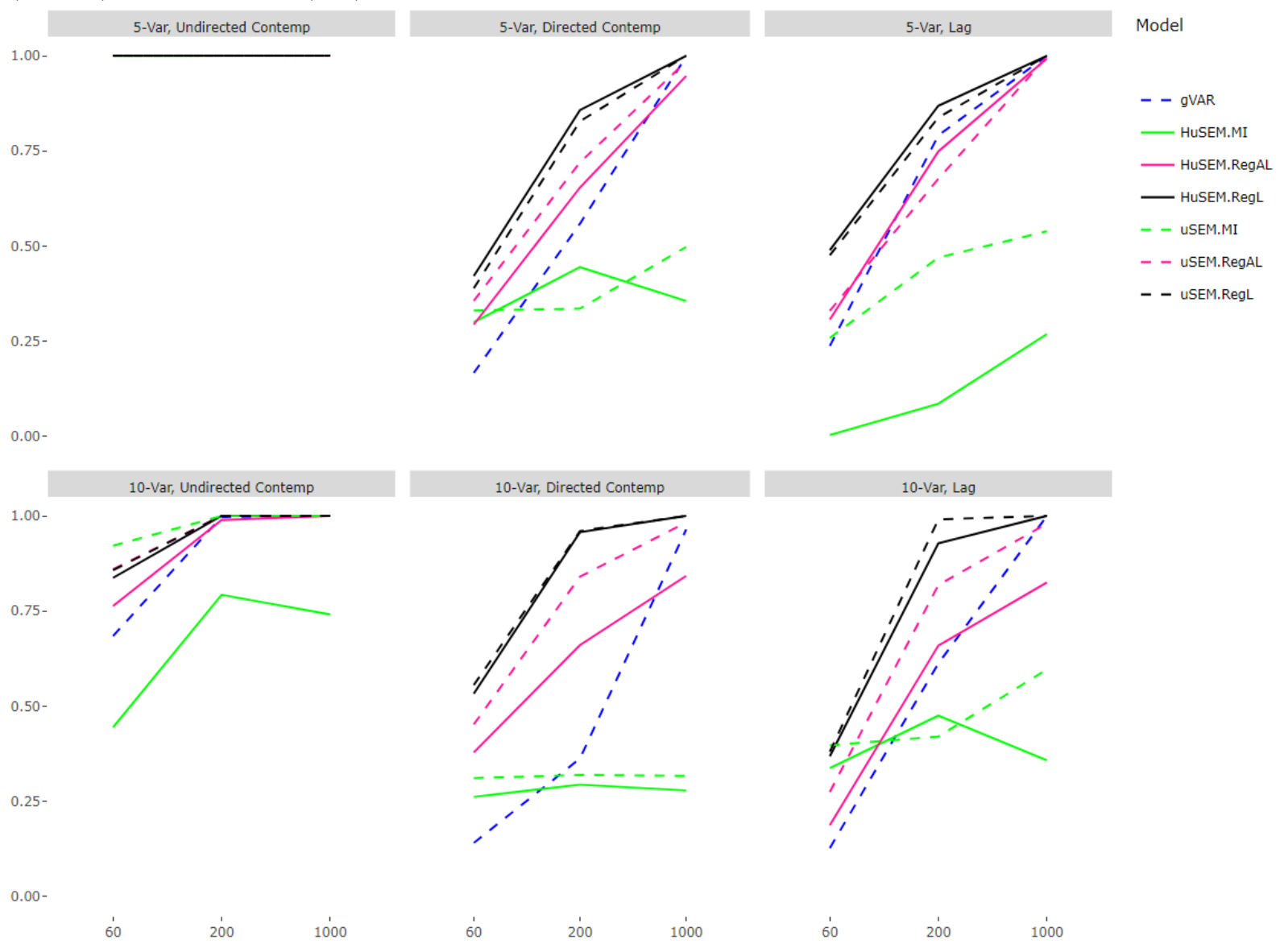

Note: In the upper left graph, the black horizontal line represents six lines stacking on top of each other. This is because all models except the HuSEM.RegAL reached a $100 \%$ recovery across the three timepoint conditions. 
$g V A R$

Bidirectional contemporaneous associations were (correctly) modeled as lag-0 undirected edges (Figure 7). The sensitivity performance of gVAR seemed to depend largely on the strength of the connection and the sample size. When this connection was the strongest of the three types of relations (left column), gVAR showed $100 \%$ sensitivity for such relation in all small-size models, and in models with 10 variables and $T=200,1000(68.5 \%$ for $\mathrm{V}=10, \mathrm{~T}$ $=60)$. gVAR with a large sample size can also guarantee a near perfect sensitivity even when the connection is weaker than directed relations (left and middle columns), but such recovery rate was below satisfactory with a smaller sample sizes, ranging from $36.3 \%$ to $79.1 \%$. A small sample size was not sufficient to recover consistently a moderate bidirectional contemporaneous relation (ranged from $12.8 \%$ to $23.8 \%$ ). These observations suggested that a marginal covariance in VAR can be represented as a partial correlation in gVAR given enough statistical power.

Figure 8.

Path Direction Sensitivity (Y-axis) for Undirected Contemporaneous Relation by Timepoint (X-axis), Strength (column), and Dimension (row)

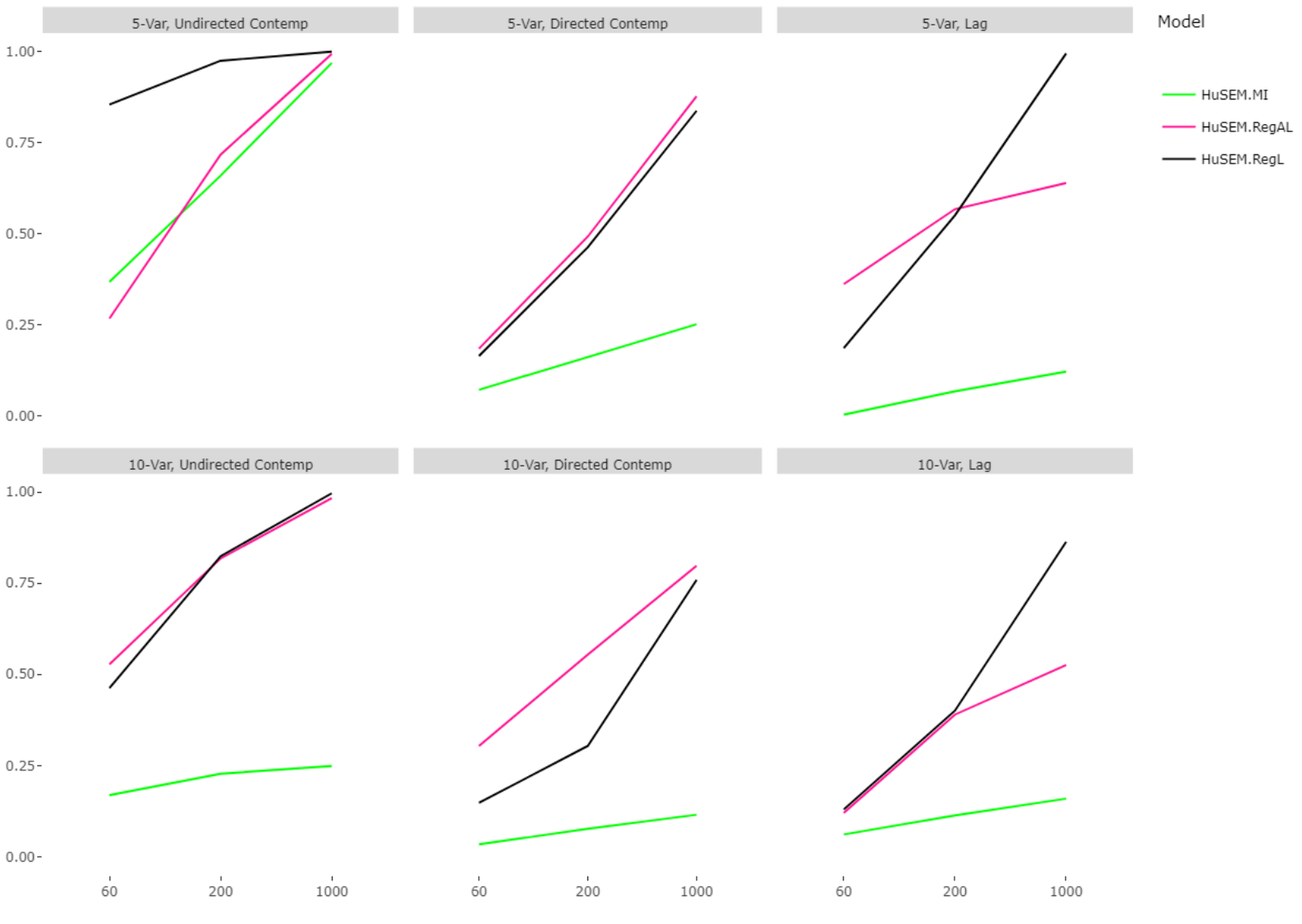

Note: Results are shown for the four out of the seven models that contain undirected contemporaneous relations; in contrast, the three uSEMs only allow directed contemporaneous paths have zero direction sensitivity for undirected contemporaneous relations. 
MI-uSEM and Reg-uSEM (with LASSO or aLASSO penalty)

Presence recovery of undirected contemporaneous relations was counted if it was recovered as a directed contemporaneous path with either direction (nonzero beta-weight). As a result, however, the direction recovery would be zero as the chance of a false positive direction was $100 \%$. The merit is that the presence of a strong relationship was not completely omitted, yet at the cost of a spurious causal evidence, i.e., unavoidable random direction imposed on the relation. The presence recovery for strong covariance contemporaneous relations was overall excellent in MI-uSEM, but sensitivity for moderate covariance relations dropped substantially. In comparison, Reg-uSEM with either penalty type exhibited a higher presence sensitivity for any undirected relations. LASSO slgihtly outperformed aLASSO.

\section{MI-huSEM and Reg-huSEM (with LASSO or aLASSO penalty)}

Much like the previous models, reg-huSEM conducted with LASSO showed perfect presence recovery for a strong undirected contemporaneous relation. This contrasts reg-huSEM with adaptive LASSO, which greatly underperformed when compared to all other approaches. When the relation was detected with adaptive LASSO, it was likely to be the correct direction (i.e., not a directed relation but rather an bidirected one). MI-huSEM was not sensitive to moderate relations and no slight improvement over MI-uSEM was observed. In addition, although covariance contemporaneous relations were incorporated in huSEM, MI-huSEM and aLASSO-huSEM still showed a high tendency to recover them as directed contemporaneous paths when such relations were strong, just as did in the constrained uSEM models. Indeed, Reg-huSEM with LASSO penalty was superior to MI-huSEM in recovering the presence of moderate undirected relations. Taken together, Reg-huSEM with LASSO penalty is uniformly the best in the recovery of the presence of covariance contemporaneous relations. When considering directionality (i.e., was it considered a undirected/bidirected relation among residuals or a directed relation among observed variables), gVAR performed better at $T=1000$ and generally matched the performance of Reg-huSEM. This provides evidence in favor of regularization approaches over stepwise procedures. 
Figure 9.

Path Sensitivity (Y-axis) for Cross-lagged Relation by Timepoint (X-axis), Strength (column), and Dimension (row)

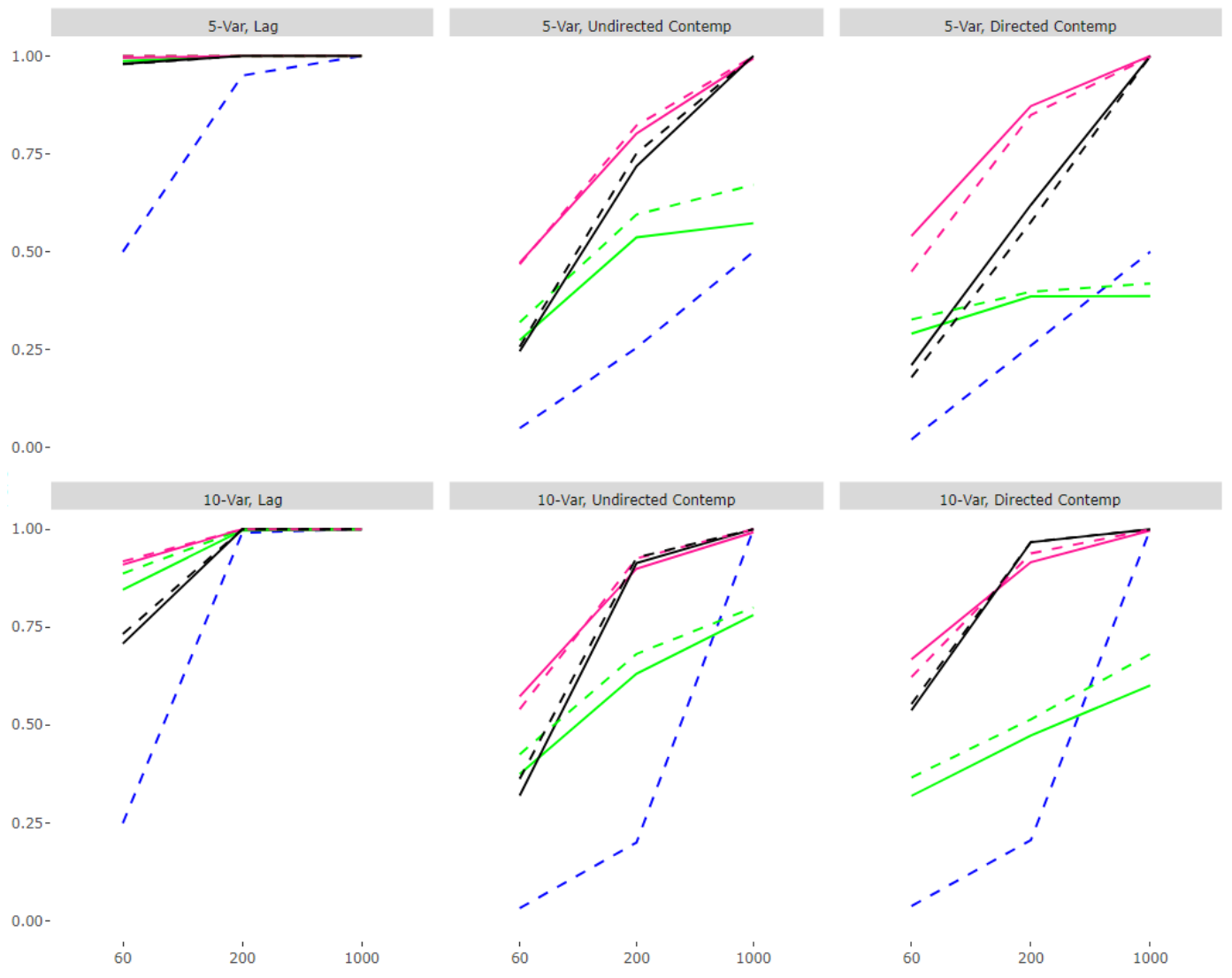

Cross-lagged effects are the only relations correctly specified in all the candidate models. As expected, the recovery was very satisfactory across models (Figure 9). Here are some observations. First, sample size was again the deciding factor for the sensitivity of a cross-lagged effect in gVAR with perfect recovery for strong cross-lagged effects or moderate ones given a large sample size when the number of variables is 10 . Second, path strength was the deciding factor for the sensitivity performance in gVAR as well as in USEM and huSEM models with MI estimation - these models showed a poor recovery for moderate cross-lagged effects even with a large sample size. Reg-uSEM and Reg-huSEM with either penalty method outperformed gVAR or MI-uSEM and MI-huSEM with respect to the recovery of moderate cross-lagged relations.

\section{Specificity}

Note that specificity in the current study refers to the ability to exclude false paths or edges that are not related with the true paths in the DGM. This means that the corresponding 
directed contemporaneous path recovered in USEM as representative of a true undirected relation, or a PCC and a PDC edge in gVAR for a true directed relation will not be counted against specificity. Under this rule, all models except gVAR showed a specificity consistently higher than $95 \%$ (close to $100 \%$ when sample size got larger), indicating their exceptional ability to remove false relations. In comparison, specificity of gVAR model was overall lower with a larger variability, ranging from $81 \%$ to $98 \%$. An interesting observation was that larger sample size was associated with poor specificity performance: all models with $\mathrm{N}=1,000$ had a specificity lower than $90 \%$ while those of models with $\mathrm{N}=60$ or 200 were mostly slightly above $90 \%$. This finding that gVAR is more likely to introduce false positive edges as sample size increases may reflect that the model is attempting to accommodate the misspecification inherent in conducting a VAR on data generated with huSEMs. Recall that gVAR recovers a strong directed contemporaneous relation as both a directed lag-1 PDC edge (for direction) and an undirected lag-0 PCC edge (for presence) at the same time. The lost in the degree of freedom as a consequence potentially brings some challenge to the estimation of the entire model, an issue that is enlarged with an increased sample size. Therefore, although we found that the sensitivity performance of gVAR improved substantially as sample size gets larger, it is at the cost of decreasing specificity.

\section{Supplemental Simulation}

As a supplemental analysis to complete the story, we generated another two sets of data using models that represented a uSEM (with only directed contemporaneous relations) and a restricted VAR (with only undirected contemporaneous relations), respectively. The goal is to investigate how the hybrid USEM (as a more general model) works when the true model is actually a more restricted one. The first data generating model (DGM(1)) is a five-variable uSEM model that contains the two directed contemporaneous relations in Figure 1, i.e., $V 1 \rightarrow V 2$ and $V 1 \rightarrow V 4$ and the lagged effect between $V 3$ and $V 4$. DGM(2) is a five-variable VAR that consists of the undirected relation $V 3 \leftrightarrow V 5$ and the lagged effect between $V 3$ and $V 4$. We used the norm sample size condition (i.e., $T=200$ ), and the same setting for path weight factor, i.e., at each time one type of the relations is strong while the others moderate. The sensitivity result was similar to those of the main simulation (see Table 3 in Appendix C).

Briefly, we found that the presence recovery for the directed paths in DGM(1) was overall good when the relation was strong. However, performance dropped substantially when the relation was weak, gVAR performed relatively the best in this regard. Reg-huSEM with LASSO, MI-uSEM, gVAR, and MI-huSEM were most reliable in recovering the direction of a directed contemporaneous relation, though the direction recovery of a moderate relation was challenging for all models. For the recovery of undirected relations in $\operatorname{DGM}(2)$, it was apparent that gVAR and Reg-huSEM with LASSO can consistently recover the presence and direction of the relations, although the chance of missing a moderate one or recovering it as a directed path in Reg-huSEMwas still nontrivial. While the other models can recover the presence well, the uSEM models cannot recover the direction (because they are modeled as directed only) yet Reg-huSEM with aLASSO or MI-huSEM have half a chance to recover the direction of a strong relation and an even lower likelihood for that of a moderate one. These two sets of results pointed to Reg-huSEM with LASSO and gVAR as the best option when models are restricted. 


\section{Empirical Example}

In this section, we illustrate the models using an empirical example. We fit the candidate models to a publicly available data set obtained from the Autism Brain Image Exchange (ABIDE; Craddock et al., n.d.; Di Martino et al., 2014) repository of fMRI data. Given that we do not know the ground truth or data generating models, we compare the models recovered by these approaches and discuss the different interpretations that result from each model choice. We also offer several precautions regarding potential issues arising from these model assumptions that researchers should be aware of when adopting one over the other.

For the processed brain data, we randomly picked two individuals from one of the sites that provide data to the ABIDE project. The number of observations for each individual was $T=246$. The data used here were collected while the participants were in a resting state, meaning that they were not involved in a task and were instructed to stare at a cross-hair. We utilized data that underwent a standard preprocessing pipeline called the Configurable Pipeline for the Analysis of Connectomes (C-PAC, http://fcp-indi.github.com) which was made publicly available by Craddock et al. (n.d.). As a result of the preprocessing steps, the data are normally distributed and weakly stationary (i.e., the mean and variance are constant) across time with cyclical trends removed.

We used time series data of five brain regions from the automated anatomical labeling (AAL) atlas (Tzourio-Mazoyer et al., 2002): the left and right Insula (I_L and I_R), right median cingulate $(\mathrm{CM})$, right precentral gyrus $\left(\mathrm{P} \_\mathrm{R}\right)$, and the left supplementary motor area (SMA). We selected the left and right Insula because bilateral brain regions typically evidence contemporaneous relations across time (e.g., Beltz \& Molenaar, 2016; Gates et al., 2017) and we wanted to see how these methods may differ in the recovery and interpretation of paths that likely will exist. The CM is an integral part of the limbic system, and thus is likely to relate to at least one of the two Insula regions as they both have been shown to be related to aspects of consciousness and higher level brain processing such as emotion processing. We would also expect that the two motor regions, the P_R and SMA, would relate to each other. We standardized the variables to account for unequal variances that are common in fMRI data. We fit five models to the $246 \times 5$ standardized time series data of the two individuals respectively: gVAR, MI-uSEM, MI-huSEM, Reg-uSEM, and Reg-huSEM. For the Reg-uSEM and Reg-huSEM, we used LASSO penalty. The aLASSO penalty was abandoned for the issue we observed from the simulation study ( $\mathrm{R}$ codes are available as supplemental materials). Figure 10 shows the result of the five models for person one, and Figure 11 for person two.

For person one, expected associations were found within the two bilateral brain regions (i.e., the left and right Insula) as well as within the motor regions (i.e., P-R and SMA) by all methods, but results differ with respect to the direction of the relation at the contemporaneous (i.e., lag-0) level or at the lag-1 level. The relations across bilateral, limbic, and motor regions also differed. For example, gVAR recovered a sparsest lag-1 network, all the five regions were contemporaneously connected at least by a second-order (i.e., through a third variable). This suggests an absence of Granger Causality from the network disclosed by gVAR. This was compared to a two-way lagged relations (indicating a mutual Granger Causality) between the two motor regions (P-R and SMA) revealed in MI-uSEM and MI-huSEM, or a one-way relation (P-R Granger caused SM) in Reg-uSEM and Reg-huSEM, plus an additional instantaneous reciprocal effect in Reg-uSEM or a undirected association in Reg-huSEM. 
Figure 10 .

Result of fMRI Study Person One

(a) gVAR

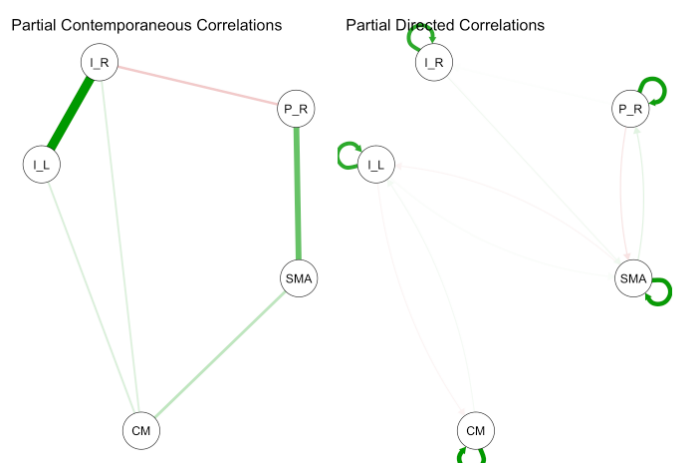

(b) MI-uSEM vs. MI-huSEM
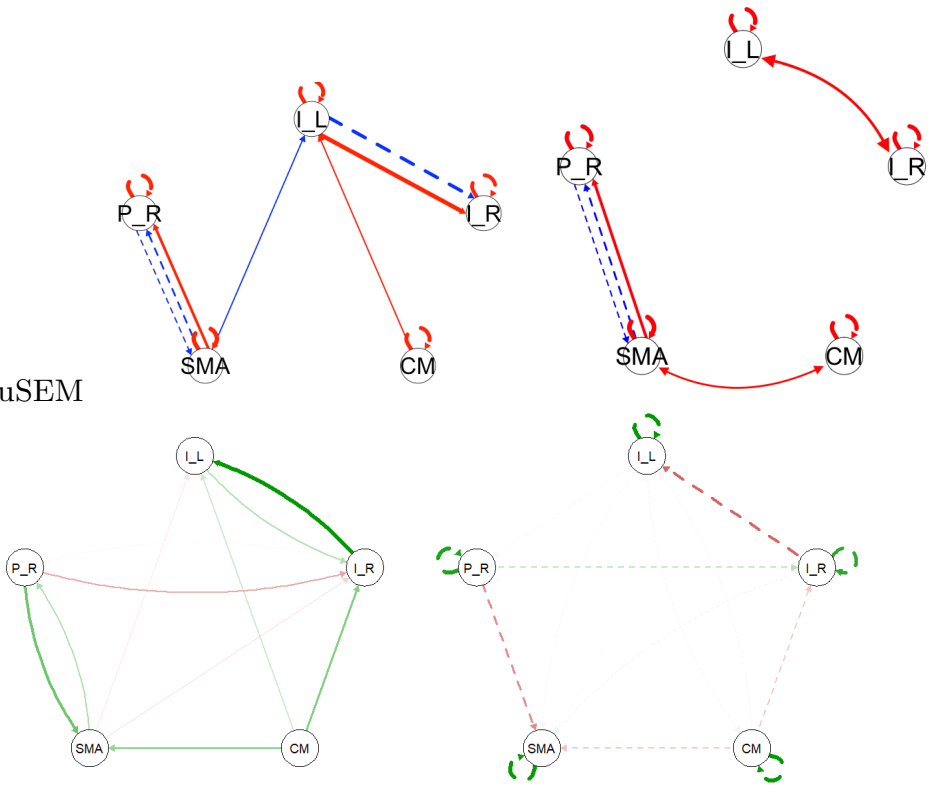

(c) Reg-uSEM

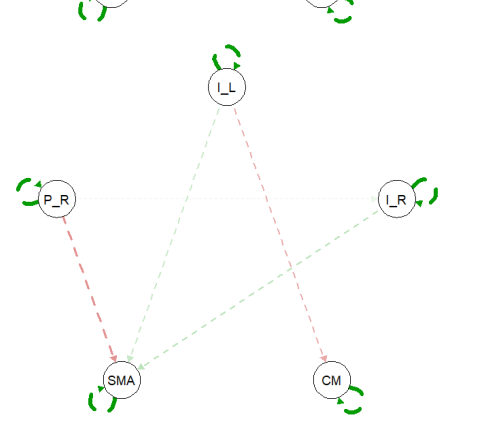

(d) Reg-huSEM

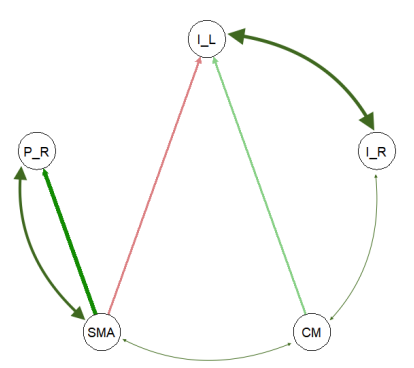

Note: 1) IL = left Insula, IR = right Insula, $\mathrm{CM}=$ right median cingulate, $\mathrm{PR}=$ right precentral gyrus, $\mathrm{SMA}=$ left supplementary motor area; 2) regsem does not provide a plotting function, we used qgraph to plot the result. Plot consists of solid lines on the left represents contemporaneous relations with two-headed curves represent covariance relations, plot with dotted lines on the right represents lagged effects. Green lines represent positive values, and red represent negative. Line width corresponds with coefficient estimate size. Plots only present $>.01$ relations, similar to the convention in programs like graphicalVAR. 
An interesting pattern showed up for the relation between the two bilateral regions across these models: while an unidirectional lagged effect was found in MI-uSEM and Reg-uSEM with an additional association on the contemporaneous level, this relation was estimated as undirected contamporaneous covariance in gVAR, MI-huSEM, and Reg-huSEM results. These together show that only the huSEM models have the capacity to recover both the Granger causality and common-cause relations that might exist simultaneously in neuroimaging data. Within the two huSEM models, MI-huSEM was sparser than Reg-huSEM, a pattern consistent to the simulation results in which we found models with MI searching procedure tend to be conservative in recovering moderate relations. Differences were also observed on the variable level in terms of the centrality or the importance of each variable in the network. For instance, the MI-uSEM and Reg-huSEM models pointed to Insula-L and SMA as central regions that showed more connections and also played a more critical role in linking (or Granger causing) the two brain clusters. From the gVAR results, Insula-L and Insula-R are equally central to the system in terms of processing, while in MI-huSEM the two clusters were by and large isolated.

Similar to person one, the overall result of person two revealed two "clusters" of these brain regions, i.e., the bilateral regions and the limbic system (CM), plus the motor regions. For person two, the two clusters were more isolated by most models. Once again, gVAR recovered a network consisted of contemporaneous connections rather than lagged relations. The MI-uSEM and Reg-uSEM further recovered the direction of these relations on both the lag- 0 and lag- 1 levels. This provided evidence for a Granger causality between SMA and P_R or CM and I_L. Additionally, a contemporaneous mediation path from CM to I_R via I_L was recovered in MI-uSEM, while a lagged one from CM to I_L via I_R was seen in Reg-uSEM. In contrast, the MI-huSEM and Reg-huSEM suggested a network with hybrid relations: while the contemporaneous relations between the pair SMA and P-R or that of CM and ILL were both undirected covariance, a directed, Granger causal relation was recovered from I_L to I_R. Once again, the hybrid models represent a network of relations that is more conservative than that of the fully directed uSEM models yet more deterministic and informative than that of a fully undirected (on the lag-0 level) gVAR model. The difference in the same pair of variables across these two participants suggests interindividual differences in their intraindividual brain networks, which further points to the need of choosing a flexible modeling framework when the ground truth is unknown in empirical settings.

We can extend this to larger network or experiment with more individual data, but these two examples demonstrate some interesting patterns already. From these results, we found that the uSEM models recovered a brain map with more frequent and directed connections, while the network recovered by gVAR is sparse in the lagged network and less informative at the level of undirected relations. The fact that gVAR model returned a sparse temporal network may largely be due to the fast-speed nature of brain activities and limitation from neuroimaging data, which further points out the importance of including directed relations on the lag-0 level to account for structural functional connectivity that are commonly seen (Friston et al., 2013; Smith, 2012). The two hybrid uSEM models represent a picture that is somewhere in between, i.e., some relations might be association by nature, while others might be causal or even mutually causing each other. Sometimes such mutual or reciprocal effects even happen at a different rate with the possibility of having a combination of signs at each level, e.g., one may have an instant positive effect on the other while the effect the other 
Figure 11.

Result of fMRI Study Person One

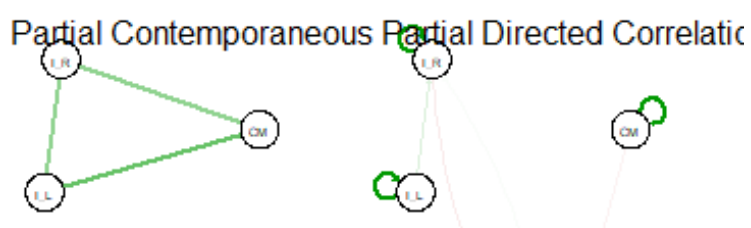

(a) gVAR
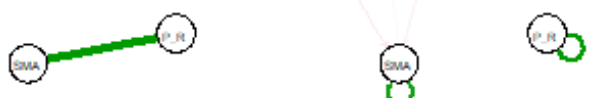

(b) MI-uSEM vs. MI-huSEM
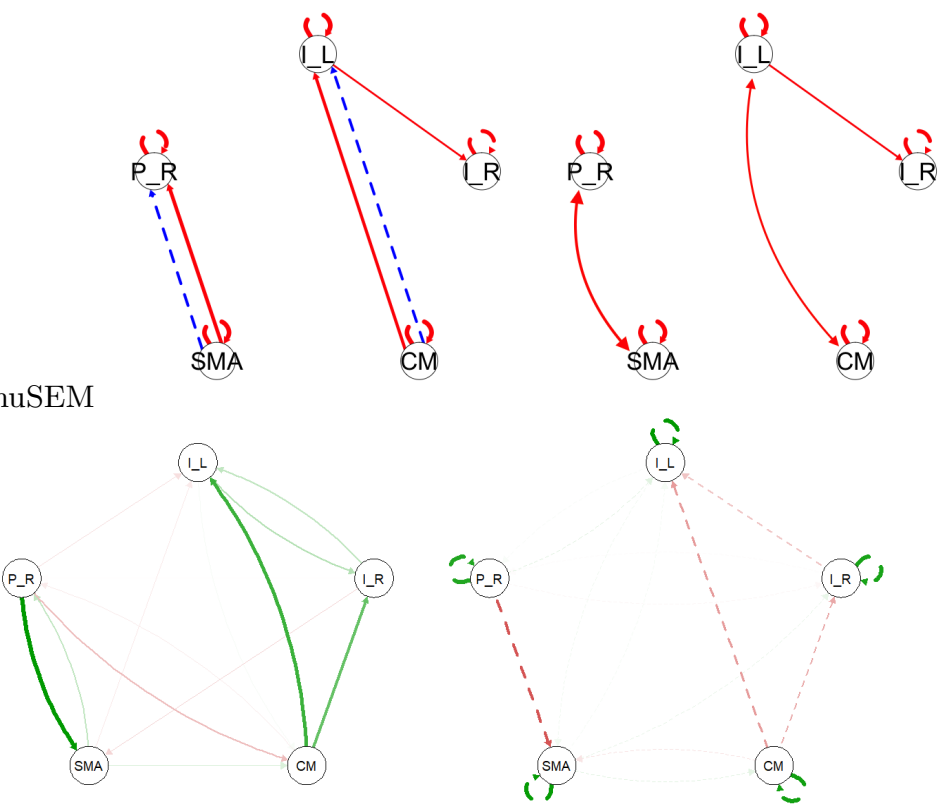

(1)

(c) Reg-uSEM

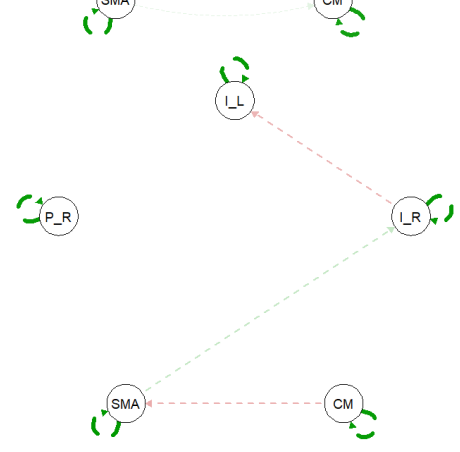

(d) Reg-huSEM
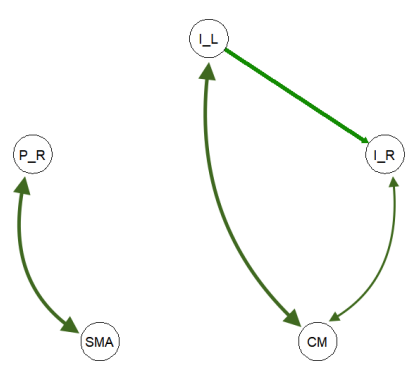

Note: 1$) \mathrm{IL}=$ left Insula, $\mathrm{IR}=$ right Insula, $\mathrm{CM}=$ right median cingulate, $\mathrm{PR}=$ right precentral gyrus, $\mathrm{SMA}=$ left supplementary motor area; 2) regsem does not provide a plotting function, we used qgraph to plot the result. Plot consists of solid lines on the left represents contemporaneous relations with two-headed curves represent covariance relations, plot with dotted lines on the right represents lagged effects. Green lines represent positive values, and red represent negative. Line width corresponds with coefficient estimate size. Plots only present $>.01$ relations, similar to the convention in programs like graphicalVAR. 
way around is negative and takes place at a slightly slower pace as a lagged effect.

The empirical example further illustrates and complements to our findings under application settings. From the neuroimaging studies, we observed similarities and differences in the networks and inferences disclosed by the these approaches. Specifically, differences from the gVAR, uSEM, and huSEM models were seen at the variable level regarding the relative importance of each variable, or at the network level with respect to the sparsity, the presence and the direction of relations and hence the interpretation in the perspective of Granger causality. Particularly, the dynamic networks revealed by uSEM or huSEM trace back to the sources of variation (e.g., SMA or CM), and point to the end (e.g., P-R or I-R, respectively). These provide us with the direction of information flows as indicative of underlying causal pathways. The importance of each construct involved is described by where it stands on the pathway. The gVAR dynamic networks, by contrast, are characterized by mutual interactions among nodes rather than the information flow in a direction. The centrality of nodes is revealed by the quantity of related existing links (often measured by some defined statistics) instead of the relative position along a flow. In fact, pathways do not exist in undirected networks, and it is meaningless to call a node of an edge the "source" or the "outcome" because they are not distinguishable. Relatedly, constructs represented by nodes relate to each other to varying degrees but are not caused by any source information from within the system. For example, P-R and SMA, are roughly the same central to the system. They are both mutually related to each other and to other nodes, but we cannot tell if they are the causes or the outcomes.

\section{DISCUSSION}

In studying person-specific dynamic models using time series data, it is possible to observe the coexistence of instantaneous causal pathways and simultaneous connections beyond lagged effect. Researchers interested in intraindividual variability should consider modeling the intraindividual variability using a general VAR representation where hybrid forms of contemporaneous relations can be estimated (e.g., Molenaar \& Lo, 2016). To overcome the limitation in the current practice for the identification and estimation of this hybrid VAR approach, we introduced a novel modeling framework, the regularized hybrid unified SEM (Reg-huSEM). The hybrid uSEM, as an extension of uSEM that had been widely used to estimate SVAR, is flexible in specifying a hybrid VAR with contemporaneous relations entering the model either as directed regression weight or as undirected covariance. We utilized regularization under the hybrid USEM framework in place of MI step-wise model search algorithm previously used by uSEM (Gates et al., 2010) and huSEM (Molenaar, 2019). Regularization provides a global, continuous model search and parameter estimation for the optimal sparse model. Our simulation validated that the sparse hybrid uSEM model obtained by regularization with LASSO penalty was the closest to the true model among all model specification and estimation methods. That is, the presence and direction of true paths of hybrid forms were recovered with the highest accuracy.This study of a person-specific model with both directed and bidirectional contemporaneous relations estimated under regularized SEM is, as far as we are aware, the first of its kind.

Two issues were addressed by our simulation study. First, our results confirmed that the hybrid uSEM is indeed superior to USEM with respect to avoiding false directed 
contemporaneous relations or graphical VAR in terms of accurately specifying the direction of structural contemporaneous relations. This conveys the message that a hybrid uSEM, rather than any restricted form of VAR, should be the starting model to fit time series data when the data generating model could potentially contain both types of relations. Specifically, graphical VAR does not consider the direction of a given contemporaneous structural relation and uSEM tends to impose a random (half chance to be false) direction to a strong covariance relation regardless of the modeling searching algorithm. It becomes even more challenging for gVAR and USEM models in recovering and specifying the correct direction of a moderate relation. Second, we found that regularization using LASSO outperformed the stepwise approach.

One may wonder why the choice of different representations of VAR models is important, given that they can be analytically transformed. The specification of directed contemporaneous relation is indeed important because while some of the VAR representations are likelihood equivalent (see Appendix A for our illustration on the model equivalency, see also Molenaar \& Lo, 2016), they differ in their interpretations. Recall that a statistical correlation or covariance represents an existence of connection whose source is exogenous to the model, and barely informs the direction of information flow. By comparison, the property of Granger causality that offers the predictability of ongoing activity in one part from that in another, is often more attractive to researchers. The concept of Granger causality is very useful to interpret directed functional connectivity when applied to neuroimaging and neurophysiological time series data such as EEG or MEG signals (Friston et al., 2013). For example, Barrett et al. (2012) used EEG to show that anaesthetic loss of consciousness Granger caused a reliable increase in cingulate cortices, extending previous "phase synchrony" obtained using undirected models (Murphy et al., 2011).

We stress that a contemporaneous directed relation is indicative of a Granger causal effect that is failed to be captured by temporal dependency due to inappropriate time scaling, which often occurs in studies like fMRI where the underlying neuronal brain activity happens much faster (on the scale of milliseconds) than what is captured via the MRI (typically on the order of seconds) and also in ecological momentary assessment studies. Recall the observation of a very sparse PCC recovered by gVAR for both individuals in our empirical fMRI example. When this is the case, an instantaneous Granger causality, if allowed, can occur when the time lag of the effect of interest is so short that it approaches infinitely to zero. Indeed, mutual causation in a dynamic system can be interpreted as a limit form of the lagged effect schemes where the time lag is reduced towards zero (Strotz \& Wold, 1960). Taking these into consideration, the inclusion of contemporaneous directed relations is essential to the completeness of potential causality.

We emphasize some major conceptual points in interpreting the directed and undirected contemporaneous networks. First, valid causal inferences can be made only from structural relations that carry casual hypotheses, such as lagged paths; whereas partial correlations or marginal correlations without causal assumptions do not correspond to a unique causal interpretation. This means that the undirected edges recovered from the lag-0 PCC network in gVAR lack the ability to accurately distinguish different causal relations or to reliably represent marginal independence. Second, not all Granger causalities can be depicted by the temporal dependencies between repeated observations and estimated as lagged relations. Particularly, the lag-1 PDC is insufficient to represent those that happen instantaneously (shorter than the measurement window), it is likely that short-term effects are 
missing from the temporal ordering information in the data. While the lag-0 PCC network can recover some remaining information as undirected partial correlations, the information about the direction (and hence the causal implication) is lost. This is when directed relations are necessary at the lag-0 level to capture the potential Granger causality relations.

Another essential contribution of our study is the development of a model search regime effective for the hybrid uSEM. Our results showed clearly that for the model search approach, regularization consistently outperformed forward-selection step-wise search with respect to reliably and accurately identified moderate relations in both the USEM and the hybrid uSEM models. MI seems to only indicate the presence and direction of strong relations; however, the inclusion or deletion of moderate connections are unlikely to cause a significant change in MI. In terms of the type of penalty method, LASSO outperformed adaptive LASSO especially for the direction recovery of structural relations. It is worth noting that although gVAR also adopted regularization to search for a sparse model, it suffers from low sensitivity performance when number of observation is small yet decreasing specificity performance as number of observation increases. This comes down to the point that a good estimation method cannot make up for unnecessary constraints in the model specification. Altogether, the LASSO-regularized hybrid uSEM model is the best combination of modeling and estimation framework for intraindividual variability analysis on multivariate time series data.

Indeed, the adoption of regularization filled a crucial methodological gap in the recovery of the uSEM family of models (Lane, 2017). As the true sparse model is almost always unknown, an exploratory search for the optimal hybrid VAR model without a priori theory is unavoidable in empirical research. The model selection process for hybrid uSEM involves not only a search for the (global) best fit in the likelihood, but perhaps also one with the Granger causality structure closest to the truth. An inappropriate model choice or a non-global solution leads to misleading causal inference. This poses a particular challenge as the search might involve statistically isomorphic models over the parameter space, i.e., likelihood-equivalent yet Granger causality-distinguishable models. The choice of recovering a contemporaneous relation between a directed and a undirected (or bidirectional) brings additional challenge to the model selection. Although the forward-selection method for uSEM model has been shown effective when applied to fMRI studies (e.g., Gates et al., 2016), it is not without issues as are all step-wise approaches. The lack of sensitivity to moderate relations found in our simulation study is not negligible. The adoption of the regularized SEM method offers hope to overcome the identification and estimation challenge in hybrid uSEM. Our simulation results validated that LASSO regularization is more effective to recover both the presence and the direction of small to moderate dynamic relations than is hybrid uSEM with MI search.

\section{Limitation and Future Direction}

There were several limitations in the present study. First, our simulation design is modest in the random simulation factors to keep a manageable scope and provide proof of concept. For example, we are fully aware that the size of network chosen is likely to be smaller than what one might encounter in time series studies. For instance, brain network studies using fMRI data is often high dimensional and might involve a network as large as 300-400 nodes (Smith et al., 2011). The choice of a small amount of variables is by and large limited by the computational speed and feasibility. The current optimization algorithm is limited both in 
the computation speed and the number of regularized variables in the model.

Second, a few assumptions are made to the data generating model as a preliminary investigation. For one, data was generated using a weakly stationary multi-normal time series, suggesting constant estimates for relations with a fixed structure. In reality, relationships might undergo some change through the passage of time. The findings should be generalized with caution, given a lack of randomness and the adoption of a simple model structure (e.g., between every pair of variables can only be an contemporaneous or a temporal connection). For two, consistent with the candidate models, a lag-1 model is assumed, higher-order lagged effect was not accounted for. Additionally, missing data was not investigated. Although missing data is usually handled by full information maximum likelihood under the assumption of missingness at random in SEM literature (Enders \& Bandalos, 2001), neither regsem or graphicalVAR programs at the time of the study has the mechanism for handling missing data.

As it does in practice, nonconvergence became another issue to consider in our study. The fact that not all the models with simulated data converged raises issues about how to best report the results. Following a common practice, we eliminated all replications that did not converge and our success rate was based on using only replications that converged. If we counted nonconverged replications as failures, then the success rates would be lower, particularly for Reg-uSEM models under those conditions when nonconvergence was relatively frequent.

There are two methodological extensions for future directions. One immediate next step is to account for between-person similarities in the model search process. The present study focused on individual-level model (one individual per model). We did not consider group-level information. Prior work has shown improvements in model search when using shared information as starting model (e.g.,Gates \& Molenaar, 2012; Varoquaux \& Craddock, 2013; Luo et al., Under Review). Another advantage of this extension is that it reduces noise due to non-meaningfull intra-individual variability, which in turn would reduce the estimation of spurious relations between variables.

The other major methodological improvement is related to the package development, such as the regularization method, optimization algorithm, etc. We are aware that it is very likely that there has already been some improvement in the program or some other newer methods that could be implemented after our study was completed. In fact, just upon completion of the current paper, we learnt that a new package called psychonetrics (Epskamp, 2020) was released, which includes a function to run gVAR with potentially better model searching algorithms and an improved performance. However, it is out of time and space for the current paper to add more comparisons. Similarly, the current study did not focus on expanding the search for other algorithms or penalty methods for Reg-uSEM and Reg-huSEM beyond the built-in options in the regsem package. Even within the package itself, there is room for improvement. For example, We believe it would be beneficial to have dual or hybrid penalization in the function for different types of parameters or those of different scales. In our case, it would be to penalize the regression type of parameters in a separate term from that of the covariance type of parameters, each with a corresponding tuning parameter. This dual penalty feature is now available for the regsem function by a collaborative work of the authors and the package team. But it is still under developed for an automatic grid search over a two-dimension tuning parameter space for an optimal combination such as the current 
fashion in function $c v_{-}$regsem for a uniform penalty, which was used in our simulation. Once this is done, the current study can be extended further. The other issue to be solved for regsem is the adoption of MLE weghts for adaptive LASSO when the model from lavvan is under-identified. For one thing, the low direction sensitivity for structural contemporaneous relations by aLASSO penalty is somewhat surprising. We suspect that this might be related with the calculation of the parameter-specific weight - each regularized parameter is scaled using the corresponding MLE estimate. The paradox is that these MLE estimates were obtained from an initial unpenalized, under-identified uSEM or huSEM model, which means they are not reliable estimates of the generative process. We plan to extend our work and generalize it to more realistic conditions in our future research with the development of more efficient regularization algorithm.

The present study, to our knowledge, is the first extension of regularization to the hybrid uSEM. The success of the application points to a promising future in exploratory SEM in this context. Just as the general trend of statistics outside the field of psychometric modeling where statistical learning methods have becoming dominate variable selection or model selection method, they might as well replace step-wise methods to be the primary model search methods for SEM in general. The major challenge, however, remains to be computational. As pointed in the recent work by Pruttiakaravanich and Songsiri (2018), the question lies in finding a powerful optimization algorithm for a model as complex as SEM. Solving the fitting function in SEM often involves nonconvex and nonlinear optimization, meaning that unique and estimable solution can only be reached under very constrained scenarios (Pruttiakaravanich \& Songsiri, 2018). But with the rapid ongoing development in contemporary computational machine learning, we are confident for the future advancement in this area. 


\section{References}

Abegaz, F., \& Wit, E. (2013). Sparse time series chain graphical models for reconstructing genetic networks. Biostatistics, $14(3), 586-599$.

Anderson, J., \& Gerbing, D. (1984). The effect of sampling error on convergence, improper solutions, and goodness-of-fit indices for maximum likelihood confirmatory factor analysis. Psychometrika, 49(2), 155-173. https://doi.org/10.1007/BF02294170

Barrett, A. B., Murphy, M., Bruno, M.-A., Noirhomme, Q., Boly, M., Laureys, S., \& Seth, A. K. (2012). Granger causality analysis of steady-state electroencephalographic signals during propofol-induced anaesthesia. PloS one, 7(1), e29072.

Beltz, A. M., \& Molenaar, P. C. (2016). Dealing with multiple solutions in structural vector autoregressive models. Multivariate behavioral research, 51(2-3), 357-373.

Bentler, P. M. (1990). Comparative fit indexes in structural models. Psychological bulletin, $107(2), 238$

Bentler, P. M., \& Bonett, D. G. (1980). Significance tests and goodness of fit in the analysis of covariance structures. Psychological bulletin, 88(3), 588.

Bollen, K. A. (1989). A new incremental fit index for general structural equation models. Sociological Methods \& Research, 17(3), 303-316.

Bringmann, L. F., Vissers, N., Wichers, M., Geschwind, N., Kuppens, P., Peeters, F., Borsboom, D., \& Tuerlinckx, F. (2013). A network approach to psychopathology: New insights into clinical longitudinal data. PloS one, 8(4), e60188.

Bringmann, L., Lemmens, L., Huibers, M., Borsboom, D., \& Tuerlinckx, F. (2015). Revealing the dynamic network structure of the beck depression inventory-ii. Psychological medicine, 45(4), 747-757.

Chen, G., Glen, D. R., Saad, Z. S., Hamilton, J. P., Thomason, M. E., Gotlib, I. H., \& Cox, R. W. (2011). Vector autoregression, structural equation modeling, and their synthesis in neuroimaging data analysis. Computers in biology and medicine, 41(12), $1142-1155$.

Chou, C.-P., \& Bentler, P. M. (1990). Model modification in covariance structure modeling: A comparison among likelihood ratio, lagrange multiplier, and wald tests. Multivariate Behavioral Research, 25(1), 115-136.

Chou, C.-P., \& Huh, J. (2012). Model modification in structural equation modeling.

Chow, S.-M., Ho, M.-h. R., Hamaker, E. L., \& Dolan, C. V. (2010). Equivalence and differences between structural equation modeling and state-space modeling techniques. Structural Equation Modeling: A Multidisciplinary Journal, 17(2), 303-332. https://doi.org/10.1080/10705511003661553 doi: 10.1080/10705511003661553

Craddock, C., Benhajali, Y., Chu, C., Chouinard, F., Evans, A., Jakab, A., Khundrakpam, B. S., Lewis, J. D., Li, Q., Milham, M., Yan, C., \& Bellec, P. (n.d.). The neuro bureau preprocessing initiative: Open sharing of preprocessed neuroimaging data and derivatives ibr / $i$. Frontiers in Neuroinformatics, (41). https://doi.org/10.3389/conf.fninf.2013.09.00041

Di Martino, A., Yan, C.-G., Li, Q., Denio, E., Castellanos, F. X., Alaerts, K., Anderson, J. S., Assaf, M., Bookheimer, S. Y., Dapretto, M., et al. (2014). The autism brain imaging data exchange: Towards a large-scale evaluation of the intrinsic brain architecture in autism. Molecular psychiatry, 19(6), 659-667. 
Eichler, M. (2005). A graphical approach for evaluating effective connectivity in neural systems. Philosophical Transactions of the Royal Society B: Biological Sciences, 360 (1457), 953-967.

Enders, C. K., \& Bandalos, D. L. (2001). The relative performance of full information maximum likelihood estimation for missing data in structural equation models. Structural equation modeling, 8(3), 430-457.

Epskamp, S. (2018). Graphicalvar: Graphical var for experience sampling data [R package version 0.2.2]. https://CRAN.R-project.org/package=graphicalVAR

Epskamp, S. (2020). Psychonetrics: Structural equation modeling and confirmatory network analysis. http://psychonetrics.org/

Epskamp, S., \& Fried, E. I. (2016). A primer on estimating regularized psychological networks. arXiv preprint arXiv:160\%.0136\%.

Epskamp, S., Waldorp, L. J., Mõttus, R., \& Borsboom, D. (2018). The gaussian graphical model in cross-sectional and time-series data. Multivariate Behavioral Research, 53(4), $453-480$.

Fisher, A. J. (2015). Toward a dynamic model of psychological assessment: Implications for personalized care. Journal of consulting and clinical psychology, 83(4), 825.

Fisher, A. J., \& Boswell, J. F. (2016). Enhancing the personalization of psychotherapy with dynamic assessment and modeling. Assessment, 23(4), 496-506.

Friedman, J., Hastie, T., \& Tibshirani, R. (2019). Glasso: Graphical lasso: Estimation of gaussian graphical models [R package version 1.11].

https://CRAN.R-project.org/package=glasso

Friedman, J., Hastie, T., \& Tibshirani, R. (2008). Sparse inverse covariance estimation with the graphical lasso. Biostatistics, 9(3), 432-441.

Friston, K., Moran, R., \& Seth, A. K. (2013). Analysing connectivity with granger causality and dynamic causal modelling. Current opinion in neurobiology, 23(2), 172-178.

Gates, K. M., Henry, T., Steinley, D., \& Fair, D. A. (2016). A monte carlo evaluation of weighted community detection algorithms. Frontiers in Neuroinformatics, 10, 45.

Gates, K. M., Lane, S. T., Varangis, E., Giovanello, K., \& Guiskewicz, K. (2017). Unsupervised classification during time-series model building. Multivariate behavioral research, 52(2), 129-148.

Gates, K. M., \& Molenaar, P. C. (2012). Group search algorithm recovers effective connectivity maps for individuals in homogeneous and heterogeneous samples. NeuroImage, 63(1), $310-319$.

Gates, K. M., Molenaar, P. C., Hillary, F. G., Ram, N., \& Rovine, M. J. (2010). Automatic search for fmri connectivity mapping: An alternative to granger causality testing using formal equivalences among sem path modeling, var, and unified sem. NeuroImage, $50(3), 1118-1125$.

Gates, K. M., Molenaar, P. C., Hillary, F. G., \& Slobounov, S. (2011). Extended unified sem approach for modeling event-related fmri data. NeuroImage, 54(2), 1151-1158.

Geweke, J. (1982). Measurement of linear dependence and feedback between multiple time series. Journal of the American statistical association, 77(378), 304-313.

Granger, C. W. (1969). Investigating causal relations by econometric models and cross-spectral methods. Econometrica: Journal of the Econometric Society, 424-438.

Hamaker, E. L., Dolan, C. V., \& Molenaar, P. C. M. (2002). On the nature of sem estimates of arma parameters. Structural Equation Modeling: A Multidisciplinary Journal, 9(3), 
347-368. https://doi.org/10.1207/S15328007SEM0903\{\-\}3

doi: 10.1207/S15328007SEM0903_3

Hamilton, J. D. (1994). Time series analysis (Vol. 2). Princeton university press Princeton, NJ.

Hastie, T., Tibshirani, R., \& Wainwright, M. (2015). Statistical learning with sparsity: The lasso and generalizations. Chapman; Hall/CRC.

Hillary, F. G., Roman, C. A., Venkatesan, U., Rajtmajer, S. M., Bajo, R., \& Castellanos, N. D. (2015). Hyperconnectivity is a fundamental response to neurological disruption. Neuropsychology, 29(1), 59.

Hoerl, A. E., \& Kennard, R. W. (1970). Ridge regression: Biased estimation for nonorthogonal problems. Technometrics, 12(1), 55-67.

Huang, P.-H. (2019). Lslx: Semi-confirmatory structural equation modeling via penalized likelihood. Journal of Statistical Software.

Huang, P.-H., Chen, H., \& Weng, L.-J. (2017). A penalized likelihood method for structural equation modeling. psychometrika, 82 (2), 329-354.

Jacobucci, R. (2017). Regsem: Regularized structural equation modeling.

Jacobucci, R., Grimm, K. J., Brandmaier, A. M., Serang, S., Kievit, R. A., \& Scharf, F. (2019). Regsem: Regularized structural equation modeling [ $\mathrm{R}$ package version 1.3.9]. https://CRAN.R-project.org/package=regsem

Jacobucci, R., Grimm, K. J., \& McArdle, J. J. (2016). Regularized structural equation modeling. Structural equation modeling: a multidisciplinary journal, 23(4), 555-566.

Jöreskog, K. G., \& Sörbom, D. (1981). Lisrel 5: Analysis of linear structural relationships by maximum likelihood and least squares methods;[user's guide]. University of Uppsala.

Jöreskog, K. G., \& Sörbom, D. (1986). Lisrel vi: Analysis of linear structural relationships by maximum likelihood, instrumental variables, and least squares methods. Scientific Software.

Kaplan, D. (1988). The impact of specification error on the estimation, testing, and improvement of structural equation models. Multivariate Behavioral Research, 23(1), 69-86.

Kim, J., Zhu, W., Chang, L., Bentler, P. M., \& Ernst, T. (2007). Unified structural equation modeling approach for the analysis of multisubject, multivariate functional mri data. Human Brain Mapping, 28(2), 85-93.

Lane, S. (2017). Regularized structural equation modeling for individual-level directed functional connectivity.

Lane, S., Gates, K., Fisher, Z., Arizmendi, C., Molenaar, P., Hallquist, M., Pike, H., Henry, T., Duffy, K., Luo, L., \& Beltz, A. (2019). Gimme: Group iterative multiple model estimation [R package version 0.6-1]. https://github.com/GatesLab/gimme/

Lane, S. T., Gates, K. M., Pike, H. K., Beltz, A. M., \& Wright, A. G. (2019). Uncovering general, shared, and unique temporal patterns in ambulatory assessment data. Psychological Methods, 24(1), 54.

Lauritzen, S. L. (1996). Graphical models (Vol. 17). Clarendon Press.

Luo, L., Gates, Z. F., Fisher, Arizmendi, C., Molenaar, P. C. M., \& Beltz, K. M., Adriene Gates. (Under Review). Estimating both directed and bidirectional contemporaneous relations in time series data using hybrid-gimme. Psychological Methods.

Lütkepohl, H. (2005). New introduction to multiple time series analysis. Springer Science \& Business Media. 
MacCallum, R. (1986). Specification searches in covariance structure modeling. Psychological bulletin, $100(1), 107$.

MacCallum, R. C., Roznowski, M., \& Necowitz, L. B. (1992). Model modifications in covariance structure analysis: The problem of capitalization on chance. Psychological bulletin, $111(3), 490$.

Meehl, P. E. (1990). Why summaries of research on psychological theories are often uninterpretable. Psychological reports, 66(1), 195-244.

Molenaar, P. C. (2017). Equivalent dynamic models. Multivariate behavioral research, 52(2), $242-258$.

Molenaar, P. C. (2019). Granger causality testing with intensive longitudinal data. Prevention Science, 20(3), 442-451.

Molenaar, P. C., \& Lo, L. L. (2016). Alternative forms of granger causality, heterogeneity and non-stationarity. W., Wiedermann, A. von Eye,(Eds.), Statistics and causality: Methods for applied empirical research, 205-230.

Murphy, M., Bruno, M.-A., Riedner, B. A., Boveroux, P., Noirhomme, Q., Landsness, E. C., Brichant, J.-F., Phillips, C., Massimini, M., Laureys, S., et al. (2011). Propofol anesthesia and sleep: A high-density eeg study. Sleep, 34(3), 283-291.

Myin-Germeys, I., Oorschot, M., Collip, D., Lataster, J., Delespaul, P., \& Van Os, J. (2009). Experience sampling research in psychopathology: Opening the black box of daily life. Psychological medicine, 39(9), 1533-1547.

Nichols, T. T., Gates, K. M., Molenaar, P. C., \& Wilson, S. J. (2014). Greater bold activity but more efficient connectivity is associated with better cognitive performance within a sample of nicotine-deprived smokers. Addiction biology, 19(5), 931-940.

Price, R. B., Lane, S., Gates, K., Kraynak, T. E., Horner, M. S., Thase, M. E., \& Siegle, G. J. (2017). Parsing heterogeneity in the brain connectivity of depressed and healthy adults during positive mood. Biological psychiatry, 81(4), 347-357.

Pruttiakaravanich, A., \& Songsiri, J. (2018). Convex formulation for regularized estimation of structural equation models.

R Core Team. (2019). R: A language and environment for statistical computing. R Foundation for Statistical Computing. Vienna, Austria. https://www.R-project.org/

Ram, N., \& Gerstorf, D. (2009). Time-structured and net intraindividual variability: Tools for examining the development of dynamic characteristics and processes. Psychology and aging, $24(4), 778$.

Rosseel, Y. (2012). lavaan: An R package for structural equation modeling. Journal of Statistical Software, 48(2), 1-36. http://www.jstatsoft.org/v48/i02/

Rothman, A. J., Levina, E., \& Zhu, J. (2010). Sparse multivariate regression with covariance estimation. Journal of Computational and Graphical Statistics, 19(4), 947-962.

Shapiro, M. D., \& Watson, M. W. (1988). Sources of business cycle fluctuations (Working Paper No. 2589). National Bureau of Economic Research. https://doi.org/10.3386/w2589

Shumway, R. H., \& Stoffer, D. S. (2017). Time series analysis and its applications: With $r$ examples. Springer.

Sims, C. A. (1981). An autoregressive index model for the u.s., 1948-1975.

Smith, S. M. (2012). The future of fmri connectivity. Neuroimage, 62(2), 1257-1266. 
Smith, S. M., Miller, K. L., Salimi-Khorshidi, G., Webster, M., Beckmann, C. F., Nichols, T. E., Ramsey, J. D., \& Woolrich, M. W. (2011). Network modelling methods for fmri. Neuroimage, 54(2), 875-891.

Steiger, J. H. (1990). Structural model evaluation and modification: An interval estimation approach. Multivariate behavioral research, 25(2), 173-180.

Tibshirani, R. (1996). Regression shrinkage and selection via the lasso. Journal of the Royal Statistical Society: Series B (Methodological), 58(1), 267-288.

Tzourio-Mazoyer, N., Landeau, B., Papathanassiou, D., Crivello, F., Etard, O., Delcroix, N., Mazoyer, B., \& Joliot, M. (2002). Automated anatomical labeling of activations in spm using a macroscopic anatomical parcellation of the mni mri single-subject brain. NeuroImage, 15(1), 273-289. https://doi.org/https://doi.org/10.1006/nimg.2001.0978

Varoquaux, G., \& Craddock, R. C. (2013). Learning and comparing functional connectomes across subjects. NeuroImage, 80, 405-415.

Weigard, A., Lane, S., Gates, K., \& Beltz, A. (Under Review). The influence of autoregressive relation strength and search strategy on directionality recovery in gimme.

Wigman, J., Van Os, J., Borsboom, D., Wardenaar, K., Epskamp, S., Klippel, A., Viechtbauer, W., Myin-Germeys, I., Wichers, M., et al. (2015). Exploring the underlying structure of mental disorders: Cross-diagnostic differences and similarities from a network perspective using both a top-down and a bottom-up approach. Psychological medicine, 45(11), 2375-2387.

Wild, B., Eichler, M., Friederich, H.-C., Hartmann, M., Zipfel, S., \& Herzog, W. (2010). A graphical vector autoregressive modelling approach to the analysis of electronic diary data. BMC medical research methodology, 10(1), 28.

Wright, A. G., Beltz, A. M., Gates, K. M., Molenaar, P., \& Simms, L. J. (2015). Examining the dynamic structure of daily internalizing and externalizing behavior at multiple levels of analysis. Frontiers in psychology, 6, 1914.

Yang, J., Gates, K. M., Molenaar, P., \& Li, P. (2015). Neural changes underlying successful second language word learning: An fmri study. Journal of Neurolinguistics, 33, 29-49.

Zou, H., \& Hastie, T. (n.d.). Tibshirani., r.,(2004). Sparse Principal Component Analysis, Technical Report, Statistics Department, Stanford University.

Zou, H. (2006). The adaptive lasso and its oracle properties. Journal of the American statistical association, 101 (476), 1418-1429. 


\section{Appendices}

\section{Appendix A: Model Equivalency}

We present here a transformation from an huSEM to an equivalent VAR. To start we provide the transformation from Equation 3 in the text:

$$
Y_{t}=(I-A)^{-1} \Phi^{*} Y_{t-1}+(I-A)^{-1} \zeta_{t}
$$

where $I$ is a $p x p$ identity matrix and all other values are as before. Note here that $\Phi^{*}$ indicates the lagged matrix obtained in USEM. It follows that the transformed $\Psi$ matrix becomes: $\Theta=(I-A)^{-1} \Psi(I-A)^{-1 \prime}$. Following this transformation, we obtain an equivalent VAR representation (Equation 2). Note that $\Phi=(I-A)^{-1} \Phi^{*}$ is the estimates one would expect from VAR analyses of this model. Here we use a numerical example to explicate the different values. Take the following data generating matrices for a hybrid uSEM:

$$
A=\left[\begin{array}{ccc}
0 & 0 & 0 \\
.4 & 0 & 0 \\
0 & 0 & 0
\end{array}\right], \Phi^{*}=\left[\begin{array}{ccc}
.5 & 0 & 0 \\
0 & .6 & 0 \\
0 & 0 & .2
\end{array}\right], \Psi^{*}=\left[\begin{array}{ccc}
.7 & 0 & .5 \\
0 & .7 & 0 \\
.5 & 0 & .7
\end{array}\right]
$$

Following transformation the matrices become:

$$
\Phi=\left[\begin{array}{lll}
.5 & 0 & 0 \\
.2 & .6 & 0 \\
0 & 0 & .2
\end{array}\right], \Theta=\left[\begin{array}{ccc}
1 & .4 & .5 \\
.4 & 1.16 & .2 \\
.5 & .2 & 1
\end{array}\right]
$$

As can be seen by this toy example, a contemporaneous covariance emerges among variables 2 and $3: \Theta_{2,3}=0.4$. When the variances of the errors are 1 , the covariance value of residuals between two given variables in a transformed VAR model will equal that of the corresponding contemporaneous coefficient in the $\boldsymbol{A}$ matrix of a huSEM. When the variance is less than one, this value will be smaller than the contemporanous path; if it is larger, this value is higher for the covariance than for the directed value. Additionally, a lagged relation between variables 2 and $3 \Phi_{2,3}=.2$ now exists in this transformed version. Since the diagonal of $\Phi$ will always be less than one for stability of the process to hold (one assumption of these time series) then the cross-lagged value in the transformed VAR will always be lower in absolute value than the value for the coefficient between the same two variables in original the $\boldsymbol{A}$ matrix. Code is provided in the online supplement for simulating data and replicating these results. 


\section{Appendix B: Convergence Result}

TABLE 2.

Rate of Convergence for the models using regsem

\begin{tabular}{ccccccc}
\hline \multirow{2}{*}{ Model Size } & Factors & \multicolumn{5}{c}{ Models } \\
Strongest Path & No. of Timepoint & RL-uSEM & RA-uSEM RL-huSEM RA-huSEM \\
\hline 5-Variable model & Directed & $\mathrm{N}=60$ & .999 & .999 & .997 & 1 \\
& Directed & $\mathrm{N}=200$ & .993 & 1 & .994 & 1 \\
& Directed & $\mathrm{N}=1000$ & .999 & .998 & .993 & 1 \\
& lag & $\mathrm{N}=60$ & 1 & 1 & .999 & .999 \\
& lag & $\mathrm{N}=200$ & 1 & 1 & .998 & .999 \\
& lag & $\mathrm{N}=1000$ & 1 & 1 & .999 & .999 \\
& Undirected & $\mathrm{N}=60$ & .997 & 1 & 1 & .998 \\
& Undirected & $\mathrm{N}=200$ & .994 & .997 & .999 & 1 \\
& Undirected & $\mathrm{N}=1000$ & 1 & .995 & .999 & 1 \\
& Directed & $\mathrm{N}=60$ & .945 & .170 & .904 & .894 \\
& Directed & $\mathrm{N}=200$ & .995 & .255 & .992 & .960 \\
& Directed & $\mathrm{N}=1000$ & .998 & .998 & .992 & .978 \\
& lag & $\mathrm{N}=60$ & .986 & .301 & .951 & .922 \\
& lag & $\mathrm{N}=200$ & .464 & .567 & .994 & .976 \\
& lag & $\mathrm{N}=1000$ & .998 & .997 & .997 & .976 \\
& Undirected & $\mathrm{N}=60$ & .956 & .956 & .909 & .898 \\
& Undirected & $\mathrm{N}=200$ & .987 & .992 & .978 & .957 \\
& Undirected & $\mathrm{N}=1000$ & .997 & .997 & .986 & .976
\end{tabular}

Nonconvergence occurs when the estimation algorithm fails to arrive at values which meet prescribed minimum criteria under the default settings of the program within a set number of iterations (Anderson \& Gerbing, 1984). Since model misspecification is a possible source of nonconvergence, the low convergence rate of Reg-uSEM might result from a number of reasons, one of which might be the omission of the covariance parameters for the contemporaneous variables. This leads to a failure to return a function of estimable parameters that reasonably approximates the variance-covariance structure of the variables provided by the data. 


\section{Appendix C: Supplemental Result}

TABLE 3.

Sensitivity Result of Supplemental Simulation

\begin{tabular}{lllllll}
\hline Models & \multicolumn{3}{c}{ DGM = uSEM } & \multicolumn{3}{c}{ DGM = VAR } \\
& $\begin{array}{l}\text { directed } \\
\text { (path) }\end{array}$ & $\begin{array}{l}\text { directed } \\
\text { (direction) }\end{array}$ & lag & undirected $\begin{array}{l}\text { undirected } \\
\text { (path) }\end{array}$ & lag \\
\hline RL-huSEM & $.948 / .421$ & $.939 / .289$ & $1 / .578$ & $1 / .73$ & $.972 / .407$ & $1 / .971$ \\
RA-huSEM & $.999 / .571$ & $.761 / .034$ & $.983 / .821$ & $.951 / .483$ & $.476 / .229$ & $.783 / .702$ \\
RL-uSEM & $.867 / .210$ & $.714 / .123$ & $.907 / .46$ & $1 / .686$ & $\mathrm{n} / \mathrm{a}$ & $1 / .968$ \\
RA-uSEM & $.580 / .423$ & $.337 / .108$ & $.899 / .743$ & $.993 / .428$ & $\mathrm{n} / \mathrm{a}$ & $1 / .758$ \\
MI-uSEM & $1 / .211$ & $.907 / .211$ & $1 / .323$ & $1 / .010$ & $\mathrm{n} / \mathrm{a}$ & $1 / .479$ \\
MI-huSEM & $1 / .223$ & $.948 / .210$ & $1 / .453$ & $1 / .320$ & $.576 / .172$ & $1 / .524$ \\
gVAR & $1 / .667$ & $* .894 / .181$ & $1 / .534$ & $1 / .708$ & $1 / .708$ & $1 / .577$
\end{tabular}

Note: path $=$ path recovery, direction $=$ direction recovery; each block shows the $\%$ of recovery with strong versus moderate path weight; *direction recovery of a directed contemporaneous path refers to a lagged effect between the variables with a correct direction. 